Wake Effects on Drift in Two-Dimensional Inviscid Incompressible Flows 


\title{
WAKE EFFECTS ON DRIFT IN TWO-DIMENSIONAL INVISCID INCOMPRESSIBLE FLOWS
}

\author{
BY \\ SERGEI MELKOUMIAN, H.B.Sc.
}

\begin{abstract}
A THESIS
SUBMITTED TO THE SCHOOL OF COMPUTATIONAL SCIENCE \& ENGINEERING AND THE SCHOOL OF GRADUATE STUDIES

OF MCMASTER UNIVERSITY
\end{abstract}

IN PARTIAL FULFILMENT OF THE REQUIREMENTS

FOR THE DEGREE OF

MASTER OF SCIENCE

(c) Copyright by Sergei Melkoumian, December, 2014

All Rights Reserved 
Master of Science (2014)

(School of Computational Science \& Engineering)
McMaster University

Hamilton, Ontario, Canada

TITLE:

Wake Effects on Drift in Two-Dimensional Inviscid Incompressible Flows

AUTHOR:

Sergei Melkoumian

H.B.Sc., (Physics)

University of Toronto, Toronto, Ontario

SUPERVISOR: $\quad$ Dr. Bartosz Protas

NUMBER OF PAGES: xii, 65 
To my parents, Ivetta and Aleksandr. 


\section{Abstract}

This investigation analyzes the effect of vortex wakes on the Lagrangian displacement of particles induced by the passage of an obstacle in a two-dimensional incompressible, inviscid fluid such that the flow is potential and time-independent in a suitable frame of reference. In addition to the trajectories of individual particles, we also study their drift and the corresponding total drift areas in the Föppl and Kirchhoff potential flow models. Our findings, which are obtained numerically and in some regimes are also supported by asymptotic analysis, are compared to the wakeless potential flow which serves as a reference. We show that in the presence of the Föppl vortex wake some of the particles follow more complicated trajectories featuring a second loop. The appearance of an additional stagnation point in the Föppl flow is identified as a source of this effect. It is also demonstrated that, while the total drift area increases with the size of the wake for large vortex strengths, it is actually decreased for small circulation values. On the other hand, the Kirchhoff flow model is shown to have an unbounded total drift area. By providing a systematic account of the wake

effects on the drift, the results of this study will allow for more accurate modeling of hydrodynamic stirring. 


\section{Acknowledgements}

First and foremost, I would like to thank my supervisor, Dr. Bartosz Protas, for all of his support throughout this research endeavour and for having the confidence to offer me this opportunity. His mentorship has allowed me to grow both academically and personally, and for this I would like to extend my sincere gratitude. I very much look forward to our future collaboration! I would also like to thank the members of my examination committee, Dr. Nicholas Kevlahan and Dr. Stephen Tullis, for all of their input and recommendations about my thesis. In addition, I would like to acknowledge the School of Computational Science and Engineering as well as the Mathematics and Statistics department at McMaster University which I consider to be a great work environment, full of many genuine and friendly people. Finally, I am deeply indebted to my family and friends for their continuous support. 


\section{Notation}

$\begin{array}{ll}\text { Symbol } & \text { Description } \\ \Omega & \text { Fluid domain } \\ \partial \Omega & \text { Domain boundary } \\ \boldsymbol{\nabla} & \text { Vector differential operator } \\ \frac{D}{D t} & \text { Material derivative } \\ \mathbf{n} & \text { Unit normal vector } \\ \mathbf{x} & \text { A point in } \Omega \\ t & \text { Time } \\ \mathbf{u} & \text { Fluid velocity } \\ p & \text { Fluid pressure } \\ \rho & \text { Fluid density } \\ \mathbf{b} & \text { Body forces on the fluid } \\ \mathbf{U} & \text { Object velocity } \\ \mathbf{a} & \text { Object acceleration } \\ \mathbf{F} & \text { Force on the object } \\ \boldsymbol{\omega} & \text { Vorticity } \\ \omega & \text { Vorticity pseudo-scalar }\end{array}$




\begin{tabular}{ll}
$\Gamma_{\mathcal{C}}$ & Circulation \\
$V$ & Complex velocity \\
$W$ & Complex potential \\
$\phi$ & Scalar potential \\
$\phi$ & Vector of unit potentials \\
$\psi$ & Streamfunction \\
$U$ & Flow velocity \\
$a$ & Cylinder radius \\
$\Gamma$ & Vortex strength \\
$\xi$ & Particle drift \\
$D$ & Total drift area \\
$M$ & Added mass \\
$A$ & Added mass tensor \\
$B$ & Body area \\
$\mathcal{K}$ & Complete elliptic integral of the first kind \\
$\mathcal{E}$ & Complete elliptic integral of the second kind \\
$k$ & Elliptic modulus \\
$u$ & Incomplete elliptic integral of the first kind \\
$\mathcal{E}$ & Incomplete elliptic integral of the second kind \\
$\mathrm{dn}$ & Jacobi elliptic function \\
\hline
\end{tabular}




\section{Contents}

Abstract $\quad$ iv

Acknowledgements $\quad$ v

Notation vi vi

1 Introduction 1

1.1 Euler System . . . . . . . . . . . . . . . . . 4

1.2 Potential Flow . . . . . . . . . . . . . . . . . . 6

1.3 Potential Flow Examples . . . . . . . . . . . . . . . . . . 8

1.4 Drift and Wakes . . . . . . . . . . . . . . . . . 13

2 Model Problems $\quad 15$

2.1 Wakeless Potential Flow . . . . . . . . . . . . . . . 16

2.2 Föppl Flow . . . . . . . . . . . . . . . . . . . 16

2.3 Kirchhoff Flow . . . . . . . . . . . . . . . . . . . . . . . 17

3 Drift: Definition and Calculation 23

3.1 Individual Particle Drift . . . . . . . . . . . . . . 23

3.2 Total Drift Area . . . . . . . . . . . . . . . . . . . 24 
3.3 Analytical Lagrangian Trajectories and Drift in the Wakeless Potential

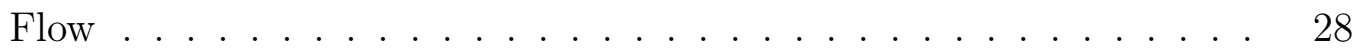

4 Results $\quad 36$

4.1 Numerical Computation of Particle Trajectories, Drift and Total Drift Area in the Föppl and Kirchhoff Flows . . . . . . . . . . . 36

4.2 Comparison of Particle Trajectories, Drift and Total Drift Area in Flows with Different Wake Models . . . . . . . . . . . . . . . . . 41

4.3 Discussion . . . . . . . . . . . . . . . . . . . . . . . . . . . . 48

5 Asymptotic Analysis $\quad 51$

6 Conclusions and Outlook $\quad 54$

$\begin{array}{ll}\text { A Added Mass } & 57\end{array}$ 


\section{List of Figures}

1.1 Schematic of the problem indicating representative particle trajectories and the coordinate systems used. The body is translated from left to right during an infinite time. The solid looping curves indicate the fluid particles' trajectories. The dashed lines indicate the initial and final resting positions of a line of particles that are initially vertically

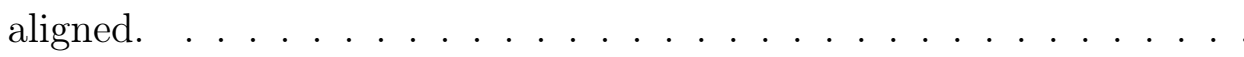

1.2 The flow around a corner ( $z$-plane) being mapped to uniform flow (zeta-plane) via the conformal map $\zeta(z)=z^{\frac{\pi}{\alpha}}, \alpha=\pi / 2 \ldots \ldots . . .11$

1.3 Level sets for potential (thin black solid line) and streamfunction (thick blue solid line) in the case of a point vortex (a), dipole (b) and corner

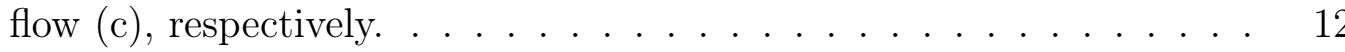

2.1 Streamlines (left column) and velocity field (right column) of the wakeless potential flow (top row), Föppl flow with $\Gamma=8.84$ (middle row) and in the Kirchhoff flow (bottom row). . . . . . . . . . .

2.2 Streamlines (left column) and velocity fields (right column) relative to a frame of reference where the cylinder is in motion. See the caption in Figure 2.1 for a description of the model types. . . . . . . . . . 
3.1 Schematic of the angles between the cylinder and the target particle $\left(\theta^{\prime}\right)$ as well as the angle that the tangent to the particle's curvature makes with the OX axis $(\eta) \ldots \ldots \ldots \ldots \ldots$

4.1 (a) Particle trajectories corresponding to $y_{0}=2$ in the wakeless potential flow obtained numerically with RelTol $=$ AbsTol $=10^{-13}$ (symbols) and evaluated analytically using formulas (3.31) and (3.33) (solid line); (b) error between the corresponding drift $\xi_{1}(2)$, cf. (3.29), and its numerical approximation $\hat{\xi}_{1}(2)$ computed using routines ode23 (open symbols) and ode45 (filled symbols) for RelTol $=10^{-10}$ (squares), $10^{-11}$ (stars), $10^{-12}$ (circles), and $10^{-13}$ (triangles). In this example, it takes $t \approx \mathcal{O}\left(10^{16}\right)$ time units for the particle to converge to machine

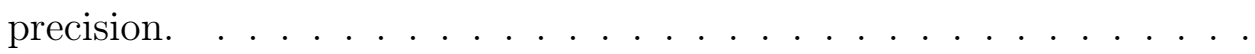

4.2 Particle trajectories for different initial conditions $\mathbf{x}_{0}=\left[0, y_{0}\right]^{T}$ in the wakeless potential flow (a), the Föppl flow with different circulations $(\mathrm{b}, \mathrm{c}, \mathrm{d})$ and the Kirchhoff flow (e). The x's represent the particle positions at unit time intervals, whereas the o's correspond to the particle positions at $t=0$, at which the cylinder, recirculation bubble for Föppl flow and the cavity for Kirchhoff flow are also indicated. . . . . . . 42

4.2 (Continued, see previous caption for details) . . . . . . . . 43

4.2 (Continued, see previous caption for details) . . . . . . . . 44 
4.3 Dependence of drift $\xi$ on (a) the vortex circulation $\Gamma$ for initial particle positions $y_{0} \in\{1.001,1.002, \ldots, 1.01,1.02, \ldots, 1.1,1.2, \ldots, 2.0\}$ (larger $y_{0}$ corresponding to lower curves), (b) the initial distance $y_{0}$ and (c) the distance $y_{\infty}$ from the flow centerline measured at infinity, cf. (3.6), for the circulation values indicated in the legend. . . . . . . . . . 46

4.4 Behavior of the velocity component $u_{x}$ following the trajectory of the particle located at $y_{0}=5$ at $t=0$ in Kirchhoff flow for large (a) negative and (b) positive times (solid line); the dashed line represents the power-law fit (4.1) with the exponent values indicated in the captions. 47

4.5 Total drift area $D$ in the Föppl flows as a function of the vortex circulation $\Gamma$ evaluated based on definition formula (3.8) (empty circles), added-mass formula (3.9) (crosses) and Taylor's theorem (3.12)-(3.13) (dashed line) . . . . . . . . . . . . . . . . . . 47

4.6 Neighborhood of the stagnation point (marked with a large dot) where the recirculation zone separatrix (dashed line) separates from the obstacle boundary (thick solid line). Particle trajectories are shown with thin solid lines with markers indicating positions at equal time intervals. 50

5.1 Dependence of drift $\xi$ on (a) the circulation $\Gamma$ for $y_{0}=1000$ and (b) the initial particle position $y_{0}$ for $\Gamma=0.38023$; solid lines represent the actual data whereas the dashed lines correspond to asymptotic formula $(5.2)$

6.1 Relative difference between the drift area in the Föppl flow $(D)$ and in wakeless potential flow $\left(D_{1}\right)$ as a function of the circulation $\Gamma$. . . 


\section{Chapter 1}

\section{Introduction}

When a body passes through an unbounded fluid, it induces a net displacement of fluid particles. The difference between the initial and final positions of a fluid particle is defined as the particle's "drift" [1], and plays an important role in characterization of the stirring occurring in multiphase flows [2] and due to swimming bodies [3]. Hereafter we will exclusively focus on flows with velocity fields stationary in a suitable steadily translating frame of reference, and will consider flows symmetric with respect to the flow centerline. Analysis of drift in time-dependent flows is more involved and some efforts in this direction have been made using methods of chaotic dynamics $[4,5]$.

Following the seminal study by Munk [6], the phenomenon of drift has recently received a lot of attention in the context of mixing in the oceans caused by swimming organisms [7]. Since the ocean is vertically stratified, the vertical motion of solid bodies such as zooplankton or larger mammals mixes the lower fluid layer which has a higher density with the higher fluid layer which has a lower density. This process causes a variation in the potential energy in the oceans which is important 
for ocean circulation and also for nutrient transport. The study [7] performed in situ measurements of swimming jellyfish through different fluid layers to validate a computational model of a moving sphere in potential flow in addition to circular and elliptical 2D swimmers in viscous flow. The empirical measurements of that study show that about $90 \%$ of the potential energy increase in the fluid can be attributed to induced drift as opposed to other causes such as turbulent wake mixing. In another study [3], the effect of drift due to multiple cylindrical or spherical swimmers in a potential fluid on a passive target particle transporting a scalar quantity such as heat, salt or a nutrient was studied. When typical physical values corresponding to krill in the ocean were considered for the swimmer, the effective diffusivity due to drift was found to be a considerable source of diffusion in the fluid. Furthermore, rough estimates showed that when the effect of viscosity was included, there was an increase in the effective diffusivity. Most of the theoretical descriptions in past studies of stirring rely on irrotational flow models used to compute or estimate the drift (an exception to this is a recent study [8] focused on the Stokesian approximation).

For a more complete theory on drift in the real world, more effects need to be considered. For instance, the recommended considerations in [3] include, "boundary layers, more realistic shape distributions for the swimming bodies, wakes, spatial correlations between swimmers, patchiness and schooling, finite correlation length of swimming, distribution of velocities, and buoyancy and stratification effects." The goal of the present contribution, the results of which have been published in [9], is to understand the effect of vortex wakes on the drift in inviscid, incompressible flows. This will be accomplished in the two-dimensional (2D) setting using a combination of careful numerical computations and mathematical analysis. The set-up of the 


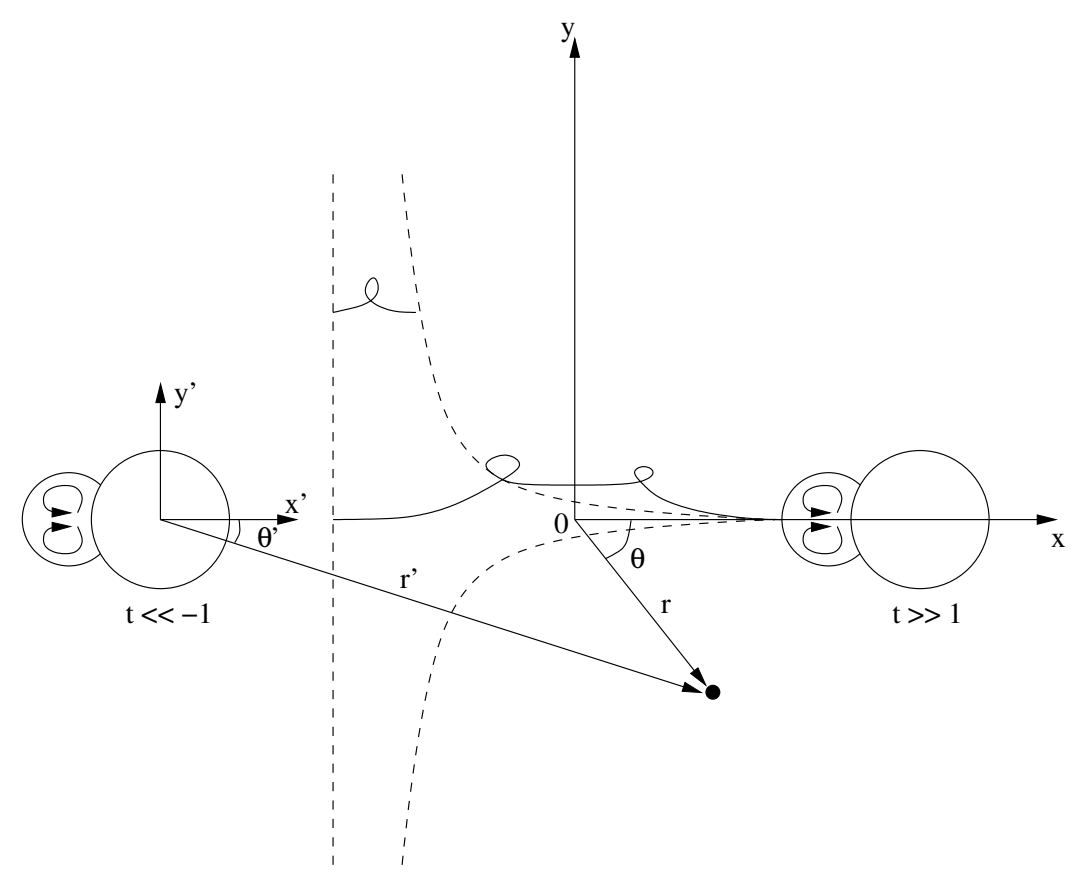

Figure 1.1: Schematic of the problem indicating representative particle trajectories and the coordinate systems used. The body is translated from left to right during an infinite time. The solid looping curves indicate the fluid particles' trajectories. The dashed lines indicate the initial and final resting positions of a line of particles that are initially vertically aligned.

problem is illustrated in Figure 1.1 with $(r, \theta)$ and $\left(r^{\prime}, \theta^{\prime}\right)$ representing, respectively, the polar coordinates in the fixed and moving frame of reference.

The structure of the thesis is as follows: We begin by introducing the theoretical framework of potential flows and providing a thorough background on drift. Then, in Chapter 2, we introduce the different vortex flows considered in our study and identify their key parameters. In Chapter 3 we give a precise definition of drift and the total drift area, explaining how these quantities can be evaluated in a given flow and rederiving some key analytical results regarding individual particle drift. Computational results are presented in Chapter 4 together with a validation of the numerical approaches, whereas their discussion and a posteriori justification via asymptotic analysis are offered in Chapter 5. Conclusions and outlook are deferred to Chapter 6. 
Finally, Appendix A gives some background on added mass which is a concept used in calculating the total drift area.

\subsection{Euler System}

In fluid mechanics there exists a framework of describing idealized flow, called the Euler system or Euler flow $[1,10]$. This system, typically derived by considerations of the conservation of mass, momentum and energy laws, is idealized in the important sense that the fluid has no viscosity, which may be a suitable approximation for the more complete Navier-Stokes equations in the case when the Reynolds number of the flow becomes large. For incompressible flows, where the rate of change of kinetic energy in a portion of fluid equals the rate at which the pressure and body forces do work, the Euler equations may be written as [10]:

$$
\begin{aligned}
\rho \frac{D \mathbf{u}}{D t} & =-\nabla p+\rho \mathbf{b}, \\
\boldsymbol{\nabla} \cdot \mathbf{u} & =0,
\end{aligned}
$$

in the fluid region we are considering, $\Omega$. A point $(x, y) \in \Omega$ will be represented as $\mathbf{x}=[x, y]^{T}, \mathbf{u}(\mathbf{x})=\left[u_{x}, u_{y}\right]^{T}$ is the fluid velocity, $p$ is its pressure, $\rho$ is a constant for the fluid density, $\mathbf{b}=\left[b_{x}, b_{y}\right]^{T}$ is any body forces acting on the fluid such as gravity. The vector differential operator is given by $\nabla:=\left[\frac{\partial}{\partial x}, \frac{\partial}{\partial y}\right]^{T}$ (the symbol ":=" defines the quantity on the left-hand side with the quantity on the right-hand side) in two dimensions. The $\frac{D}{D t}$ operator is called the material deriative and is defined as

$$
\frac{D}{D t}:=\frac{\partial}{\partial t}+\mathbf{u} \cdot \nabla
$$


In addition, for any rigid boundary $\partial \Omega$ in the fluid, we require the following boundary condition to be satisfied:

$$
\mathbf{u} \cdot \mathbf{n}=0,
$$

where $\mathbf{n}$ is the unit normal vector pointing away from the interior of the boundary and into the fluid. For example, for a circular cylinder of unit radius, $\mathbf{n}=\left[\cos \theta^{\prime}, \sin \theta^{\prime}\right]^{T}$. This boundary condition, also referred to as the no through-flow boundary condition, implies that the fluid cannot penetrate any walls. If the boundary is also in motion with velocity $\mathbf{U}=\left[U_{x}, U_{y}\right]^{T}$ in the fluid, then the boundary condition becomes

$$
\mathbf{u} \cdot \mathbf{n}=\mathbf{U} \cdot \mathbf{n} .
$$

With the given set of unknowns, $\mathbf{u}$ and $p$, these equations allow for a complete description of the flow. Note that, unlike in the Navier-Stokes equations, there is no viscosity term and that the "no-slip" boundary condition, $\mathbf{u}=\mathbf{0}$ on $\partial \Omega$, does not apply.

Besides the velocity field $\mathbf{u}(\mathbf{x}, t)$ that describes the fluid flow, there is a physical quantity called the vorticity field, $\boldsymbol{\omega}(\mathbf{x}, t)$, which is used to describe the rotation of the flow. It is defined as

$$
\boldsymbol{\omega}=\boldsymbol{\nabla} \times \mathbf{u}
$$

such that when the flow is $2 \mathrm{D}$ and in the $x y$-plane, $\boldsymbol{\omega}$ is a vector that points in the $z$-direction, thus, in the present context vorticity can be considered as a pseudo-scalar given by

$$
\omega=\frac{\partial u_{x}}{\partial y}-\frac{\partial u_{y}}{\partial x} .
$$


A more global property involving vorticity is the circulation, which may be more intuitively associated with the observed "rotation" of the fluid. If $\mathcal{C}$ is a simple, smooth, oriented closed contour of an oriented surface $\mathcal{S}$, the fluid circulation of the velocity field $\mathbf{u}$ on $\mathcal{C}$ is given, due to Stokes' theorem, by

$$
\Gamma_{\mathcal{C}}=\oint_{\mathcal{C}} \mathbf{u} \cdot d \mathbf{x}=\int_{\mathcal{S}} \mathbf{n} \cdot(\boldsymbol{\nabla} \times \mathbf{u}) d S=\int_{\mathcal{S}} \mathbf{n} \cdot \boldsymbol{\omega} d S .
$$

\subsection{Potential Flow}

An important class of fluid flows that may be used to approximate many flows in nature are called potential flows (also called "harmonic" or "irrotational"). In these flows, the velocity field can be expressed as the gradient of some scalar potential. That is,

$$
\mathbf{u}=\boldsymbol{\nabla} \phi .
$$

As a consequence of our flow being incompressible, we may write

$$
\boldsymbol{\nabla} \cdot \mathbf{u}=\nabla^{2} \phi=0
$$

so we can see that the potential $\phi$ is a harmonic function that satisfies Laplace's equation. Thus, we may take advantage of numerous results in mathematical analysis that

characterize such types of functions. In addition, these flows are called irrotational because the vorticity is zero:

$$
\boldsymbol{\omega}=\boldsymbol{\nabla} \times \mathbf{u}=\boldsymbol{\nabla} \times(\boldsymbol{\nabla} \phi)=0,
$$


almost everywhere, with the exclusion of isolated points or sheets. However, it is important to note that even for irrotational flows in $\Omega$ where the vorticity is zero, it is possible that the circulation is non-zero. Such a result would suggest that the source of vorticity giving rise to the circulation lies outside $\Omega$.

For two-dimensional flows the conditions of incompressibility and irrotationality imply that

$$
\begin{aligned}
& \frac{\partial u_{x}}{\partial x}+\frac{\partial u_{y}}{\partial y}=0 \\
& \frac{\partial u_{x}}{\partial y}-\frac{\partial u_{y}}{\partial x}=0 .
\end{aligned}
$$

Before proceeding, we will introduce the complex variable setting. A point $(x, y) \in$ $\Omega$ will be represented as a complex number $z=x+i y$, where $i:=\sqrt{-1}$. An overbar will denote complex conjugation. The fluid velocity (which will also be referred to as the complex velocity) will be denoted $V(z)=\left(u_{x}-i u_{y}\right)(z)$. Thus, the equations in (1.11) are the Cauchy-Riemann equations for the analytic function $V(z)$. The complex velocity is defined as the complex derivative of the complex potential, an analytic function given by $W(z)=(\phi+i \psi)(z)$ where $\phi$ and $\psi$ are, respectively, the scalar potential, cf. (1.8), and the streamfunction. Thus, the velocity components of the flow may be related to the potential and streamfunction in the following way:

$$
\left[u_{x}, u_{y}\right]=\left[\frac{\partial \phi}{\partial x}, \frac{\partial \phi}{\partial}\right]=\left[\frac{\partial \psi}{\partial y},-\frac{\partial \psi}{\partial x}\right] .
$$

The streamfunction $\psi(x, y)$ is particularly useful when visualizing fluid flow. When the flow is independent of time, i.e., steady, particles follow trajectories that correspond to level sets where $\psi$ is a constant. In other words, when the flow is steady 
these level sets, called streamlines of the fluid flow, are equivalent to the particle pathlines (the trajectories of individual fluid particles). When the flow is unsteady, streamlines can only be produced for a particular instance in time, thus they are less useful.

\subsection{Potential Flow Examples}

In this section we will briefly introduce some of the crucial building blocks that are necessary for describing the models that we are ultimately interested in. More specifically, the wakeless potential flow and Föppl flow in Chapter 2 rely on fundamental models described by singularities in potential flow that will be introduced here. The bulk of these simple examples are obtained from [11].

A source or a sink in two-dimensional potential flow at position $z_{0} \in \mathbb{C}$ has the complex potential

$$
W(z)=\frac{m}{2 \pi} \log \left(z-z_{0}\right),
$$

where $m \in \mathbb{R}$ is referred to as the strength of the source/sink and describes the rate of emission of volume per unit time. It is easy to verify via (1.12) that when $m>0$, this corresponds to a source where fluid is emitted away from the singularity and when $m<0$, it is a sink where fluid is being transferred into the singularity. In addition, the streamlines, coinciding with the level sets of the streamfunction $\psi$, are straight lines that extend radially from the point while the potential lines described by $\phi$ are circles.

A point vortex may be considered in analogy to a source/sink except where the streamlines and lines of constant potential are swapped. This is akin to swapping the 
real and imaginary parts of $W(z)$ which can be achieved by multiplying the complex potential by $i$. The vortex strength is written as $\Gamma \in \mathbb{R}$, and so we may write that for a point vortex with flow circulating counter-clockwise,

$$
W(z)=\frac{\Gamma}{2 \pi i} \log \left(z-z_{0}\right), \quad \Gamma>0 .
$$

In the case when the circulation is clockwise, $\Gamma<0$. See Figure 1.3a for the potential and streamline pattern. It is interesting to note that even in the presence of a point vortex, the vorticity $\omega$ of the fluid region $\Omega$ (which excludes the point $z_{0}$ ), remains zero. This is because in the case of a point vortex, vorticity is concentrated at a single point. A buoyant object placed in the vicinity of $z_{0}$ may circumnavigate the point vortex following the particle paths described by the level sets of $\psi$, however, the point vortex cannot induce a change in the object's angular velocity.

Suppose that we place a source and a sink with equal strength $|m|>0$ near to each other around the origin, with a separation distance given by $\epsilon$. Then the complex potential, given by the sum of their individual complex potentials, is

$$
\begin{aligned}
W(z) & =-\frac{m}{2 \pi} \log (z-\epsilon)+\frac{m}{2 \pi} \log (z+\epsilon) \\
& =-\frac{m}{2 \pi} \log \left[z\left(1-\frac{\epsilon}{z}\right)\right]+\frac{m}{2 \pi} \log \left[z\left(1+\frac{\epsilon}{z}\right)\right] \\
& =-\frac{m}{2 \pi} \log \left[1-\frac{\epsilon}{z}\right]+\frac{m}{2 \pi} \log \left[1+\frac{\epsilon}{z}\right] .
\end{aligned}
$$

Taking the Taylor expansion for the logarithm terms, and making the substitution 
$\mu=m \epsilon / \pi$, this becomes

$$
\begin{aligned}
W(z) & =-\frac{m}{2 \pi}\left(-\frac{\epsilon}{z}-\frac{\epsilon^{2}}{2 z^{2}}-\frac{\epsilon^{3}}{3 z^{3}}-\ldots\right)+\frac{m}{2 \pi}\left(\frac{\epsilon}{z}-\frac{\epsilon^{2}}{2 z^{2}}+\frac{\epsilon^{3}}{3 z^{3}}-\ldots\right) \\
& =\frac{\mu}{z}+\frac{\mu \epsilon^{2}}{3 z^{3}}+\ldots
\end{aligned}
$$

Then, if we take $m \rightarrow \infty$ and $\epsilon \rightarrow 0$ simultaneously such that $\mu$ remains a constant, we get that the first term in (1.16) is all that remains and that $W(z)=\mu / z$. This is the expression for the potential of a dipole or doublet of strength $\mu$. Its streamlines consist of circles of various sizes tangent to a horizontal line passing through the origin as seen in Figure 1.3b.

Uniform flow has the complex potential $W(z)=U e^{-i \alpha} z$ where $\alpha$ is the angle of the flow with respect to the $x$-axis and $U \in \mathbb{R}$ is its magnitude. We will only consider the case when $\alpha=0$. This leads us to a convenient tool when constructing potential flows in domains with certain types of boundaries:

Theorem (Milne-Thomson Circle Theorem [1]). Let a harmonic flow have complex potential $f(z)$, analytic in the domain $|z| \leq a$. If a circular cylinder of radius $a$ is placed at the origin, then the new complex potential is $W(z)=f(z)+\overline{f\left(a^{2} / \bar{z}\right)}$.

If we would like to obtain the complex potential of uniform flow past a circular cylinder with speed $U$ and radius $a$, we may apply the theorem to find that

$$
W(z)=U z+\frac{U a^{2}}{z} .
$$

Note that the second term is the equivalent of a dipole at the origin with $\mu=U a^{2}$. We will return to this model when we discuss the wakeless potential flow model in 


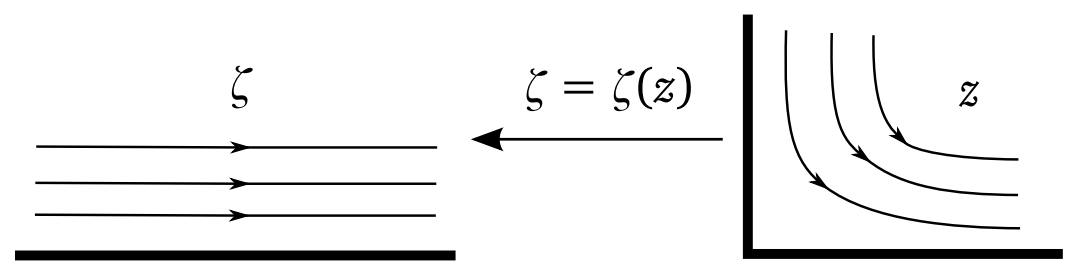

Figure 1.2: The flow around a corner ( $z$-plane) being mapped to uniform flow (zeta-plane) via the conformal map $\zeta(z)=z^{\frac{\pi}{\alpha}}, \alpha=\pi / 2$.

Chapter 2.1. For streamlines, see Figure 2.1a.

In our discussion of potential flows so far, we have been able to obtain the formulas for the complex potential with relative ease, in part, because (except in the case of the circular cylinder) we are dealing with an unbounded domain with no boundaries. There are situations, however, where solid boundaries such as walls or edges in the fluid need to be considered. As will be discussed later, these boundaries may in fact represent vortex sheets such the one that we will see in Kirchhoff flow. For these types of problems, we may use a useful tool in complex analysis called a conformal map.

Conformal maps are functions from one complex domain to another that preserve the angles between curves [12]. One useful property of conformal maps is that a harmonic function transformed from one domain to another will remain harmonic. This suggests that if we can find a conformal map from one domain or geometry to another, then we may easily evaluate the complex potential, which is the harmonic function that we are interested in.

For example, suppose we wish to determine the flow around a corner. To achieve this, we may use the complex potential for uniform flow and transform the domain, via a conformal map, to a domain with a corner in which the flow satisfies the correct boundary conditions. Thus, suppose that $z$ represents the physical domain featuring 


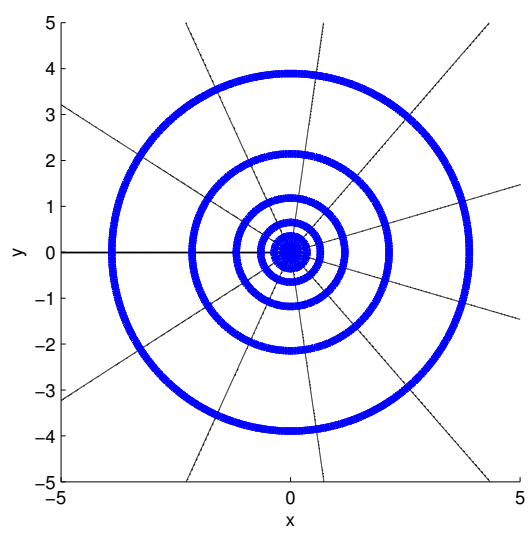

(a)

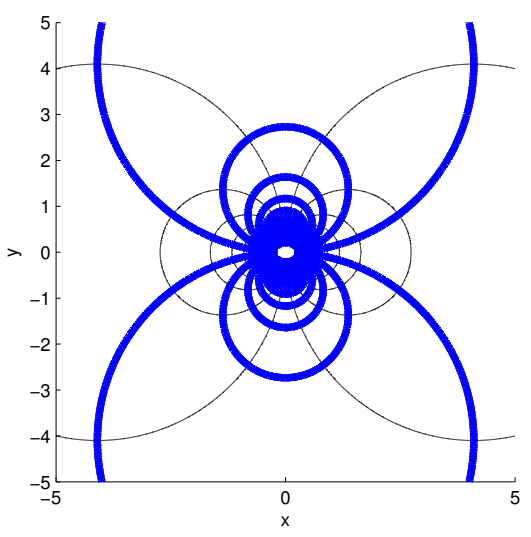

(b)

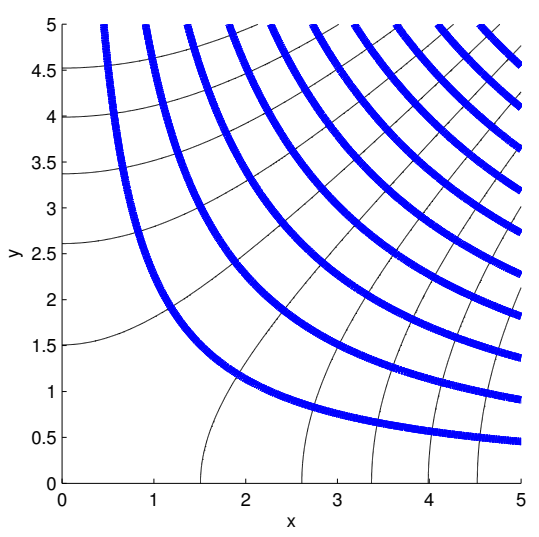

(c)

Figure 1.3: Level sets for potential (thin black solid line) and streamfunction (thick blue solid line) in the case of a point vortex (a), dipole (b) and corner flow (c), respectively.

a boundary with a corner, and $\zeta(z)$ is the mapped domain corresponding to a uniform flow where we already know that the complex potential is $W(\zeta)=\zeta$. There exists a conformal map, $\zeta(z)=z^{\frac{\pi}{\alpha}}[13]$, where $\alpha$ is the angle of the corner, which maps points in the upper half-plane to points in the first quadrant that are bounded by a boundary with a corner. Figure 1.2 shows the two boundaries and flows corresponding to the complex variables $z$ and $\zeta$. Evaluating this example for $\alpha=\pi / 2$, we find that the flow appears as shown in Figure 1.3c. 


\subsection{Drift and Wakes}

Drift has been investigated for over a century with the earliest work belonging to Maxwell [14] who showed that, when the passing object is a circular cylinder inducing a simple potential flow, then surrounding fluid particles follow trajectories in the form of "elastica" curves (a more modern account of this problem can be found in monograph [11]). For a given fluid particle, an elastica-shaped trajectory approaches a straight line parallel to the path of the moving cylinder for points far upstream and downstream, and exhibits a loop with a fore-and-aft symmetry (when the particle travels along this loop, the cylinder is underneath it). The historical origins and some other applications of elasticas are surveyed in [15]. Another major contribution to this area is due to Darwin ${ }^{1}$ who, in addition to particle trajectories, studied the problem of drift area and drift volume which are global quantities characterizing the particle drift in a given flow. Darwin's proposition [17], also referred to as a "theorem", is a key result relating the drift area or volume of a moving body to its added mass. Its utility consists in the fact that the latter quantity tends to be easier to evaluate for flows past objects with complex shape. There has been some debate $[18,19,20,21]$ concerning a rigorous proof of this result in its full generality which was centered on the evaluation method for conditionally convergent integrals. Details of this discussion are however beyond the scope of this thesis. The relation between drift volume and added mass was investigated in a controlled experiment [22] where it was found that the shape of the displaced material surface is similar to that of the inviscid case and that the added mass coefficient measured for a spherical bubble for Reynolds numbers ranging from $R e=500$ to 1000 is consistent with its value

\footnotetext{
${ }^{1}$ Charles Galton Darwin (1887-1962), an English applied mathematician, who was the grandson of Charles Darwin known for evolutionary theory [16].
} 
obtained from Darwin's theorem. Connections between the Darwinian drift and the Stokesian drift, related to the wave motion, were explored in [23].

The concept of drift was recently generalized for the case of flows induced by propagating vortices (vortex rings) in [24]. Motivated by biofluid applications, a recent study [8] investigated the effects of vortex wakes on the drift induced by simple swimmers moving in the Stokes fluid. On the other hand, recognizing that the concept of drift is idealized, in the sense that the object is assumed to travel during infinite time, corrections resulting from finite travel times were obtained leading to the definition of partial drift $[21,25]$. This quantity was studied in recent experimental investigations concerning stirring by swimmers $[24,7]$. Another related quantity is the mean squared displacement of particles which can be used to compute the effective diffusivity [3].

A trailing wake is the region of recirculating flow typically found behind a moving object in a fluid. See the classical album [26] for a visual illustration of various types of wakes. Due to the complexity of the wake region, which may indeed be turbulent, there are various models that may be used as approximations. In the present study we provide a thorough account of the effects of different vortex wakes on the drift in inviscid flows. We will focus on 2D flows, because they offer simple solutions amenable to straightforward analysis, so that closed-form results can be obtained. 


\section{Chapter 2}

\section{Model Problems}

In this Chapter we describe the three model flows we will consider in our study. In addition to the wakeless potential flow for which the questions of drift are well understood and which will serve as a reference, we will also investigate the Föppl and Kirchhoff flows which will be shown to have quite different properties. These two flows are often invoked as the possible inviscid limits of steady viscous Navier-Stokes flows [27]. For simplicity, in all three cases the cylinder radius and the free stream at infinity have unit values, $a=1$ and $U=-1$.

We will consider a circular cylinder of unit radius $(a=1)$ passing through an incompressible inviscid fluid in a $2 \mathrm{D}$ unbounded domain $\Omega$. The flow satisfies the continuity and momentum equations introduced in (1.1) and we will consider the case when $\rho=1$ and $\mathbf{b}=0$. The cylinder passes with its center along the $x$-axis from $x=-\infty$ to $x=\infty$ with constant unit speed. Hence, in the cylinder's frame of reference there is a uniform stream at infinity such that $\mathbf{u} \rightarrow U \hat{\mathbf{x}}$ as $|\mathbf{x}| \rightarrow \infty$, where $U=-1$ and $\hat{\mathbf{x}}$ is the unit vector associated with the $x$-axis. In this frame of reference, the flow is steady, i.e., $\mathbf{u}=\mathbf{u}(\mathbf{x})$. Euler system (1.1) is known to admit nonunique 
solutions and different such solutions will be discussed below.

Hereafter, without the risk of confusion, we will interchangeably use the vector and complex notation for various vector quantities. The velocity field is incompressible and irrotational, thus to reiterate some of the notation from Chapter 1.2 , it can be expressed in terms of the complex potential $W(z)=(\phi+i \psi)(z)$ as $V(z)=d W / d z$ and $V(z)=\left(u_{x}-i u_{y}\right)(z)$, where $\phi$ and $\psi$ are, respectively, the scalar potential and the streamfunction.

\subsection{Wakeless Potential Flow}

The construction of the wakeless potential model of a circular cylinder in uniform flow was discussed in Chapter 1.3 and is provided here for completeness. Making the substitutions $a=1$ and $U=-1$ in (1.17), this flow is defined by the complex potential

$$
W(z)=-\left(z+\frac{1}{z}\right)
$$

which does not involve any parameters. The flow field exhibits no separation and is characterized by symmetry with respect to both OX and OY axes. The streamline pattern and the velocity fields are illustrated in Figures 2.1a,b.

\subsection{Föppl Flow}

The Föppl vortex system [28] is a one-parameter family of solutions constructed by superimposing a pair of opposite-sign vortices with circulations $\Gamma>0$ and $-\Gamma$ located symmetrically at $z_{1}=x_{1}+i y_{1}, y_{1}>0$, and $\bar{z}_{1}$, where the overbar denotes complex conjugation, on the flow with potential (2.1). The resulting potential of the Föppl 
flow is thus

$$
W(z)=-\left(z+\frac{1}{z}\right)+\frac{\Gamma}{2 \pi i} \log \left(\frac{z-z_{1}}{z-\frac{1}{z_{1}}}\right)-\frac{\Gamma}{2 \pi i} \log \left(\frac{z-\bar{z}_{1}}{z-\frac{1}{z_{1}}}\right)
$$

The locus of equilibrium vortex locations, the so-called Föppl curve, is described by the algebraic relation

$$
r_{1}^{2}-1=2 r_{1} y_{1}
$$

where $r_{1}:=\sqrt{x_{1}^{2}+y_{1}^{2}}$. The circulation of the vortices is related to their position through

$$
\Gamma=2 \pi \frac{\left(r_{1}^{2}-1\right)\left(r_{1}^{4}-1\right)}{r_{1}^{5}}
$$

For a given circulation $\Gamma>0$, the Föppl system is a limiting solution (as the vortex area goes to zero) of a family of Euler flows with finite-area vortex patches discovered by Elcrat et al. [29] (see also [30]). The Föppl system features a closed recirculation region with size growing with $\Gamma$. As is evident from $(2.2)$, in the limit $\Gamma \rightarrow 0$ the wakeless potential flow from Chapter 2.1 is recovered. The streamline pattern and the velocity field of a representative Föppl flow are illustrated in Figures 2.1c,d. The Föppl system has been successfully employed as a model in a number of studies concerning the stability and control of separated wake flows [31, 32, 33, 34, 35].

\subsection{Kirchhoff Flow}

The Kirchhoff flow is a manifestation of the free-streamline theory of the 2D ideal flows [36]. It features an object with two free streamlines in the upper and lower half-planes that separate the external fluid from the region behind the object, called the cavity 
region, where the velocity is zero and the pressure is constant. Commonly, the object used for these types of flows is a flat plate, however, for consistency with the wakeless potential and the Föppl flows, we will consider here a 1st-order approximation of a circular cylinder presented in [37]. The Kirchhoff flow is interesting as an inviscid model, because it features an infinite wake and a finite drag.

We will first clarify the notation: variable $z$ denotes the physical plane we are interested in, where the circular cylinder is of unit radius centered at $(0,0)$ and the flow is moving from right to left, whereas variable $Z$ refers to the physical plane as used in [37], where, by the particular choice of conformal maps, the cylinder has a radius of approximately 1.77 and is centered at approximately $(1.38,0)$ with flow going in the opposite direction. We can define a map to switch between the two spaces

$$
Z(z):=-1.770434824562303 \bar{z}+1.377445608362303 .
$$

The complex potential is defined as a modified Levi-Civita transformation [37]

$$
W(\tau)=-\frac{\left(\tau-\frac{1}{\tau}\right)^{2}}{4}
$$

where $\tau=\rho e^{i \sigma}$ and $0 \leq \rho \leq 1,-\pi / 2 \leq \sigma \leq \pi / 2$. Unlike the models described in Chapter 2.1 and 2.2, potential (2.6) is not given in terms of the variable in the physical space and additional transformations are needed, so that it can be evaluated at $z$ or $Z$. An intermediate map $\zeta(\tau)$ may be used to connect the $\tau$ and $Z$ planes

$$
\zeta=\frac{d Z}{d W}
$$


and for a 1st-order approximation of a circular cylinder we have

$$
\zeta(\tau)=\frac{1+\tau}{1-\tau} e^{-0.9426 \tau+0.0191 \tau^{3}}
$$

Then, using the chain rule, we may write

$$
\frac{d Z}{d \tau}=\frac{d Z}{d W} \frac{d W}{d \tau}
$$

where the first derivative factor is (2.8) and the second can be derived from (2.6). Thus, $Z(\tau)$ can be determined up to a constant through the integration

$$
\begin{aligned}
Z(\tau) & =\int_{a}^{\tau_{0}} \frac{d Z}{d W} \frac{d W}{d \tau^{\prime}} d \tau^{\prime} \\
& =-\frac{1}{2} \int_{a}^{\tau_{0}}\left(\frac{1+\tau^{\prime}}{1-\tau^{\prime}}\right)\left(1+\frac{1}{\tau^{\prime 2}}\right)\left(\tau^{\prime}-\frac{1}{\tau^{\prime}}\right) e^{-0.9426 \tau^{\prime}+0.0191 \tau^{\prime 3}} d \tau^{\prime}
\end{aligned}
$$

where $\tau_{0}$ is an arbitrary constant. Integral (2.10) does not lend itself to analytical treatment, however, a generalized series expansion for the integrand was found up to $\mathcal{O}\left(\tau^{2}\right)$ around $\tau=0$, so that, after integration, we obtain

$$
Z(\tau)=-\frac{1}{2}\left(c_{1} \tau^{-2}+c_{2} \tau^{-1}+c_{3} \log \tau+c_{4} \tau+c_{5} \tau^{2}\right)+Z_{0}
$$

where $c_{1}=0.5, c_{2}=1.0574, c_{3}=-0.55904738, c_{4}=-0.8828122332, c_{5}=0.1113906656$ and $Z_{0}$ is some constant.

From (2.7) and (2.8), we can now compute the velocities in the $Z$-plane in terms 
of the $\tau$ variable

$$
\begin{aligned}
& u_{x}(\tau)=\Re\left(\frac{1}{\zeta(\tau)}\right), \\
& u_{y}(\tau)=-\Im\left(\frac{1}{\zeta(\tau)}\right) .
\end{aligned}
$$

Since we are interested in the flow in the direction opposite to the one in the $Z$-plane [37], we set $V(z)=\left(u_{x}-i u_{y}\right)(z)=\left(-u_{x}-i u_{y}\right)(\tau)$. In order to be able to evaluate velocities (2.12) at a given location $Z$ in the physical space, we need to invert map (2.11), i.e., find $\tau=Z^{-1}(z)$. This is done by applying Newton's method to

$$
F(\tau)=-\frac{1}{2}\left(c_{1} \tau^{-2}+c_{2} \tau^{-1}+c_{3} \log \tau+c_{4} \tau+c_{5} \tau^{2}\right)-Z=0 .
$$

Once $\tau$ is found, the velocity at the required location can be computed using (2.12). The streamlines and velocity field of the Kirchhoff flow can be seen in Figures 2.1e and 2.1f. 


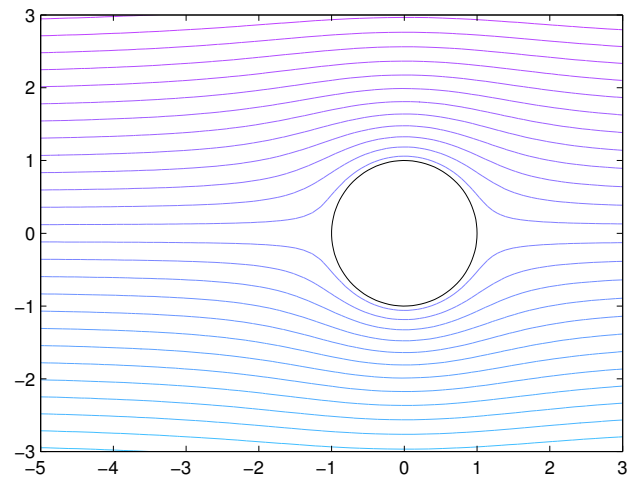

(a)

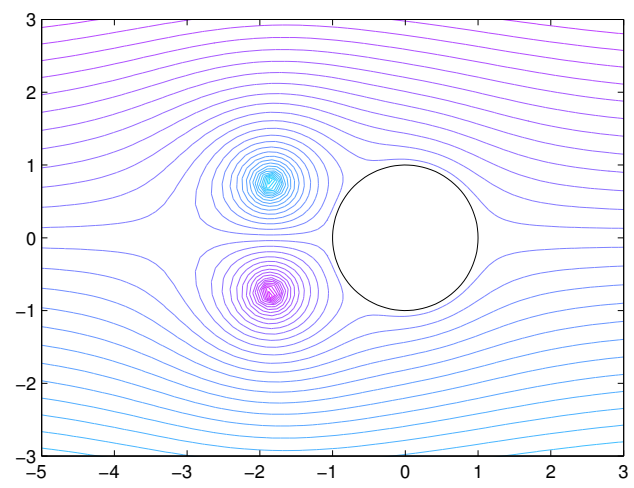

(c)

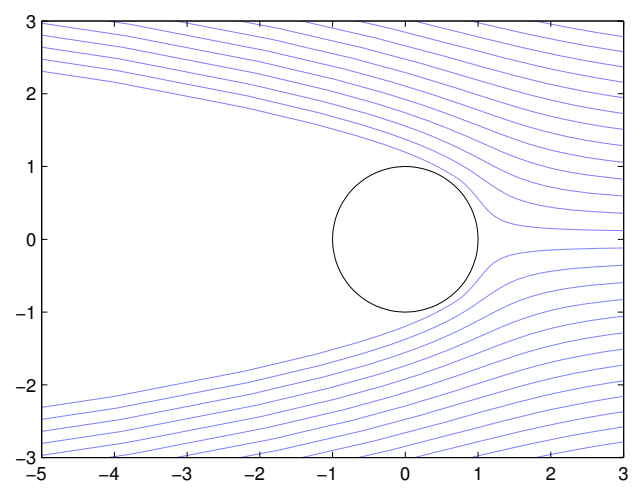

(e)

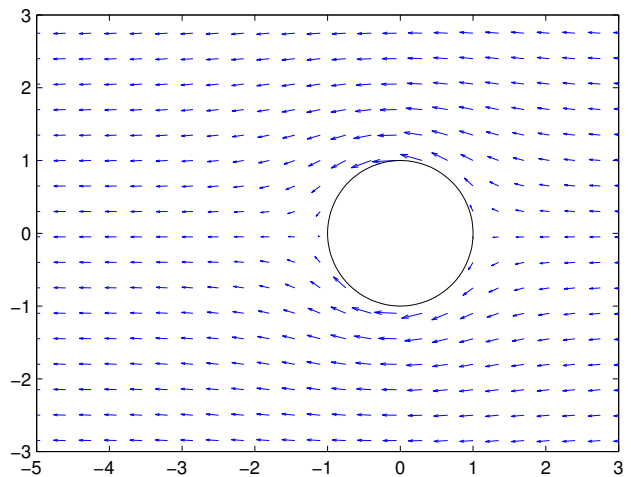

(b)

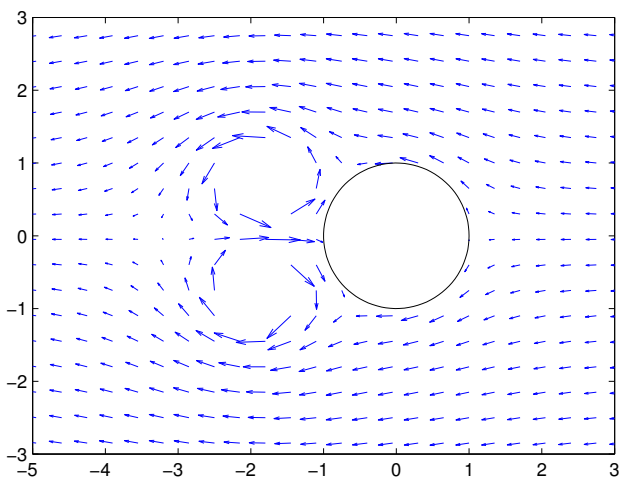

(d)

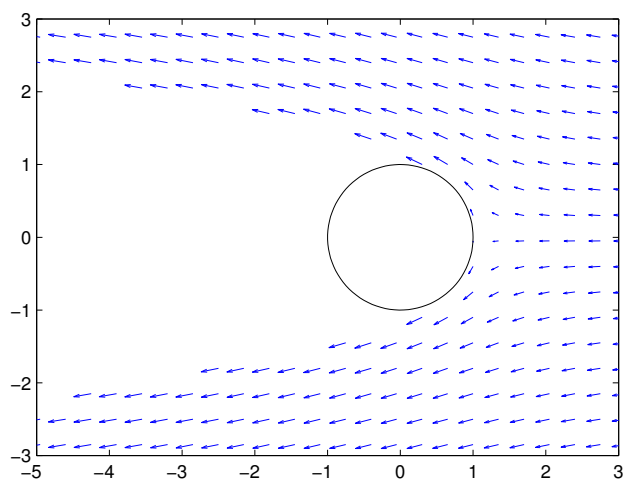

(f)

Figure 2.1: Streamlines (left column) and velocity field (right column) of the wakeless potential flow (top row), Föppl flow with $\Gamma=8.84$ (middle row) and in the Kirchhoff flow (bottom row). 


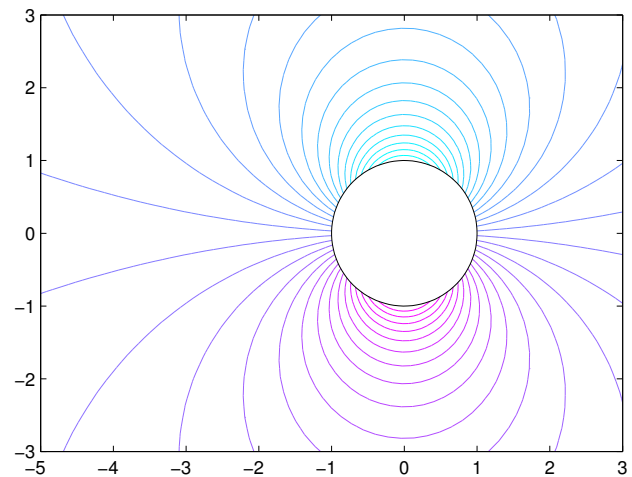

(a)

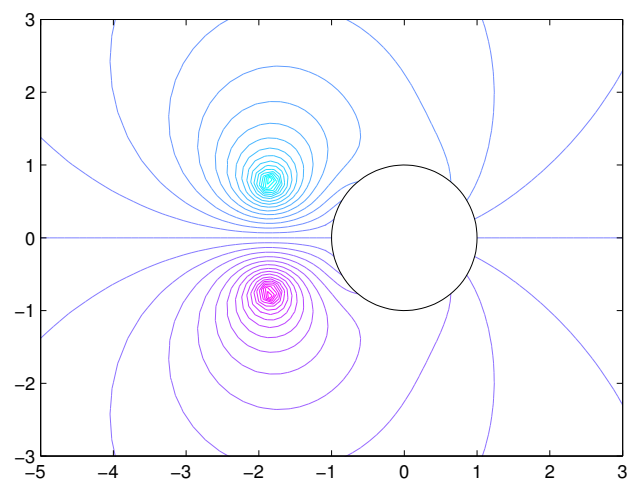

(c)

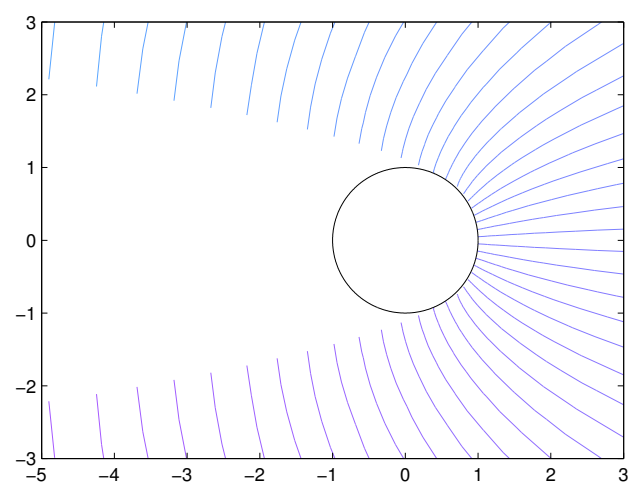

(e)

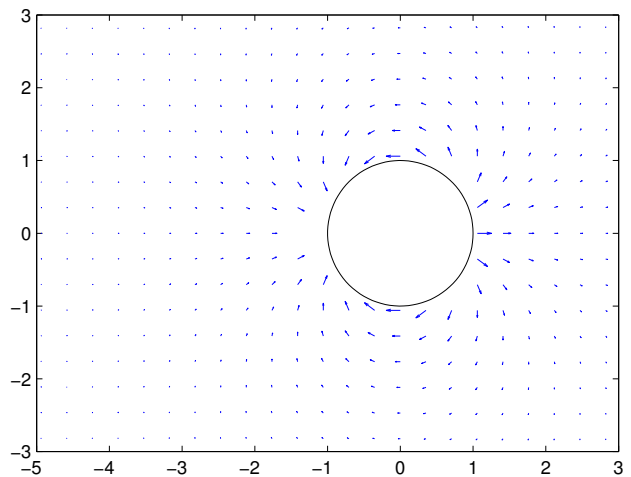

(b)

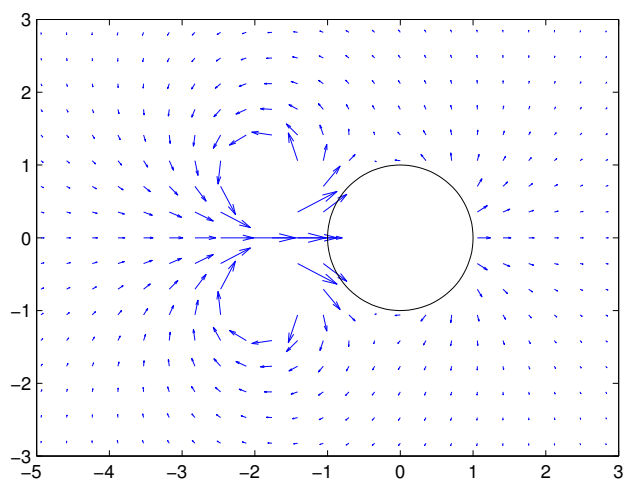

(d)

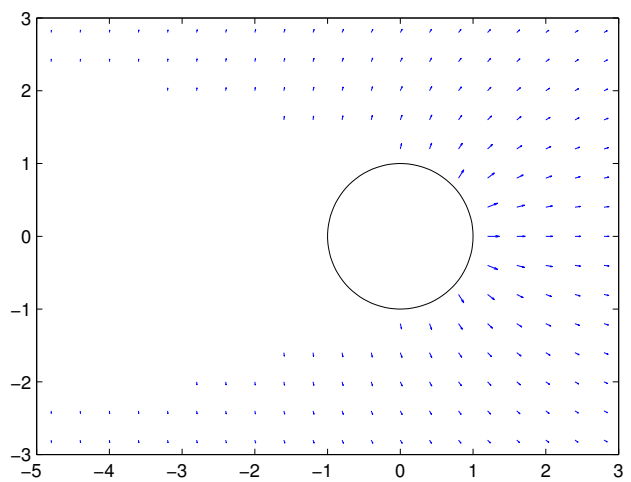

(f)

Figure 2.2: Streamlines (left column) and velocity fields (right column) relative to a frame of reference where the cylinder is in motion. See the caption in Figure 2.1 for a description of the model types. 


\section{Chapter 3}

\section{Drift: Definition and Calculation}

\subsection{Individual Particle Drift}

In much the same way that Maxwell [14] and Darwin [17] studied the problem of drift, we will consider the trajectories and drifts of individual particles in the fluid as the cylinder passes. Let the initial position of the particle at $t=0$ be $\mathbf{x}_{0}$ and $\left[x\left(t ; \mathbf{x}_{0}\right), y\left(t ; \mathbf{x}_{0}\right)\right]^{T}$ denote the corresponding particle trajectory. Then, the displacement, or drift, of the particle initially at $\mathbf{x}_{0}$ is defined as

$$
\xi\left(\mathbf{x}_{0}\right):=\int_{-\infty}^{\infty} u_{x}\left(x\left(t ; \mathbf{x}_{0}\right), y\left(t ; \mathbf{x}_{0}\right)\right) d t
$$

where the horizontal velocity component $u_{x}$ is given in the absolute frame of reference. Integral (3.1) is improper and the question of its convergence will be addressed further below. By changing the integration variable from time $t$ to the polar angle $\theta^{\prime}$ in the moving frame of reference, cf. Figure 1.1, it can be transformed to an integral (still improper) defined over a finite interval $\theta^{\prime} \in[0, \pi]$ with the bounds corresponding to 
the position of the particle in front and behind the obstacle. Rewriting the velocity in the polar coordinate system in the moving frame of reference as $\mathbf{u}=u_{r} \hat{\mathbf{r}}^{\prime}+u_{\theta} \hat{\boldsymbol{\theta}}^{\prime}$, where $\left\{\hat{\mathbf{r}}^{\prime}, \hat{\boldsymbol{\theta}}^{\prime}\right\}$ are the two unit vectors, we have for the azimuthal component

$$
u_{\theta}\left(r^{\prime}, \theta^{\prime}\right)=r^{\prime} \frac{d \theta^{\prime}}{d t} \quad \Longrightarrow \quad d t=\frac{r^{\prime} d \theta^{\prime}}{u_{\theta}\left(r^{\prime}, \theta^{\prime}\right)}
$$

and (3.1) becomes

$$
\xi\left(\mathbf{x}_{0}\right):=\int_{0}^{\pi} r^{\prime} \frac{u_{x}\left(r^{\prime}, \theta^{\prime}\right)}{u_{\theta}\left(r^{\prime}, \theta^{\prime}\right)} d \theta^{\prime}
$$

Form (3.3) is more convenient for some of the manipulations we will need to perform when deriving the drift in wakeless flow (Chapter 2.1).

\subsection{Total Drift Area}

The key quantity of interest in practical applications is the total drift area $D$ representing the integral displacement of particles initially located on a line perpendicular to the path of the obstacle at an infinite upstream distance (Figure 1.1)

$$
D:=\int_{-\infty}^{+\infty} \xi(\psi) d \psi=2 \int_{0}^{\infty} \xi\left(y_{\infty}\right) d y_{\infty}
$$

where $y_{\infty}$ is the transverse coordinate of the particle's position when $t \rightarrow-\infty$ (with a slight abuse of notation, $\xi$ may be equivalently considered a function of $\mathbf{x}_{0}, y_{\infty}$ or $\psi$ ). The two integrals in (3.4) are equal, because $\psi \rightarrow y_{\infty}$ as $x \rightarrow \infty$. The total drift area $D$ involves two nested improper integrals (in expressions (3.1) and (3.4)). Whether this quantity is actually well-defined has been the subject of a debate $[18,19,20,21]$ with the conclusion that this is indeed the case, provided the order of integration is as 
used here, i.e., first with respect to the streamwise coordinate and then with respect to the transverse coordinate. On the other hand, reversing the order of integration will result in a conditionally convergent expression.

There are two ways to evaluate the total drift area $D$. First, we can use a suitably transformed definition of formula (3.4) combined with the particle displacement given in (3.1). From the practical point of view, the most convenient way to evaluate the improper integral (3.1) is to set the particle positions $\mathbf{x}_{0}$ at $t=0$ and then obtain the trajectories by integrating the system

$$
\frac{d \mathbf{x}(t)}{d t}=\mathbf{u}(\mathbf{x}(t)), \quad \mathbf{x}(0)=\mathbf{x}_{0}
$$

forward and backward in time, i.e., for $t \rightarrow \pm \infty$, for different $\mathbf{x}_{0}$. Since the initial particle positions in formula (3.4) are given at infinity, they need to be transformed to positions with finite streamwise locations, e.g., $\mathbf{x}_{0}=\left[0, y_{0}\right]^{T}$. Since for a particle on a given streamline, $\psi$ is constant and equal to some $C$, we have

$$
C=\psi\left(0, y_{0}\right)=\lim _{x \rightarrow \infty} \psi\left(x, y_{\infty}\right)=y_{\infty} .
$$

Defining $g\left(y_{0}\right):=\psi\left(0, y_{0}\right)=y_{\infty}$ as the map between the $y$-coordinates of the particle at $x=0$ and at $x=\infty$, we obtain

$$
\frac{d y_{\infty}}{d y_{0}}=g^{\prime}\left(y_{0}\right)
$$

so that (3.4) becomes

$$
D=2 \int_{1}^{\infty} \xi\left(g\left(y_{0}\right)\right) g^{\prime}\left(y_{0}\right) d y_{0},
$$


where the lower bound is now set to unity, because the particle on the streamline with $\psi=0$ has the coordinate $y_{0}=1$ at $x_{0}=0$. We note that function $g\left(y_{0}\right)$ will be different for different solutions of Euler system (1.1).

The second method to evaluate the total drift area is to use Darwin's theorem [17] which stipulates that $D=M$, where $M$ is the added mass, and the fluid density is assumed equal to the unity. See Appendix A for a discussion on added mass. For our problem, the added mass is given by a line integral over the contour $\mathcal{C}$ which is the boundary of the largest region with closed streamlines

$$
M=\oint_{\mathcal{C}} \phi n_{x} d s,
$$

where $n_{x}$ is the $x$-component of the unit normal vector. In addition to the boundary of the obstacle, contour $\mathcal{C}$ also comprises the boundary of the recirculation region, if it is present in the flow. The reason is that, in obtaining relation (3.9), the divergence theorem cannot be applied on regions where singularities (point vortices) are present.

Alternatively, one can bypass evaluation of integral (3.9) by the application of Taylor's added mass theorem [38]. If we consider the union of our cylinder and the recirculation region as a single "body" $\mathcal{B}$ in motion, this theorem allows us to compute the added mass in terms of the singularities within this region. Suppose that our composite body contains $P$ sources and sinks with locations $z_{i}$ and strength $m_{i}$. In addition, it contains $Q$ doublets (or dipoles) with strength $\mu_{j}$ and continuously distributed sources and sinks with area density defined by $\sigma$. Then for irrotational 
flows, the generalized form of the added mass given in [39] is

$$
A_{\alpha 1}+B_{\alpha 1}+i\left(A_{\alpha 2}+B_{\alpha 2}\right)=2 \pi \rho\left[\int_{\mathcal{B}} \sigma_{\alpha} z d A+\sum_{i=1}^{P} m_{i \alpha} z_{i}+\sum_{j=1}^{Q} \mu_{j \alpha}\right], \quad \alpha=1,2,
$$

where $A$ is the added mass tensor and $B$ a tensor representing the mass of the displaced fluid per unit area of the body with entries given by

$$
B_{\alpha \beta}=\rho \oint_{\mathcal{C}} x_{\beta} n_{\alpha} d s, \quad \alpha, \beta=1,2
$$

in which $x_{1}=x, x_{2}=y, u_{1}=u_{x}, u_{2}=u_{y}$. For our problem, formula (3.10) simplifies quite significantly. In particular, since we are considering rectilinear motion in the $x$-direction of a body symmetric with the OX axis, we need only consider the element of the added mass tensor with $\alpha, \beta=1$ so we may take the real part of (3.10) and drop these indices. Further, as there are no continuous sources or sinks and $\rho=1$, we get for the added mass (now writing $A=M$ )

$$
M=2 \pi \Re\left[\sum_{i=1}^{P} m_{i} z_{i}+\sum_{j=1}^{Q} \mu_{j}\right]-B
$$

In addition, since $d s$ is a infinitesimal distance along the body, we have $n_{x} d s=d y$. Thus, $B$ can be simplified and interpreted as the area of the cylinder augmented by the area of the wake

$$
B=\oint_{\mathcal{C}} x n_{x} d s=\oint_{\mathcal{C}} x(y) d y .
$$

We remark that relation (3.12) can be interpreted as consisting of two parts: a "universal" part represented by the first term involving only the far-field expansion of the 
velocity field induced by the obstacle together with its vortex system and a second part characterizing the specific flow and represented by $B$. An analogous decomposition of the total drift area was obtained in [8] for a swimmer in the Stokes flow. While all three approaches, involving definition formula (3.4), added-mass relation (3.9) and Taylor's theorem (3.12)-(3.13), are equivalent as far as the evaluation of the total drift area is concerned, the first one offers additional insights in the form of the particle trajectories responsible for the observed drift.

\subsection{Analytical Lagrangian Trajectories and Drift in the Wakeless Potential Flow}

These classical results, recalled here for completeness, were derived by Maxwell [14] and were also surveyed in [11]. A key relation, which makes this problem analytically tractable, allows one to express the radial coordinate of the particle in the cylinder's frame of reference $r^{\prime}$ in terms of its azimuthal angle $\theta^{\prime}$ with the streamfunction $\psi$ used

as a parameter. In other words, the expression for the streamfunction for uniform flow past a circular cylinder

$$
\psi=r^{\prime} \sin \theta^{\prime}\left(1-\frac{a^{2}}{r^{\prime 2}}\right),
$$

can be rearranged to give

$$
r^{\prime}\left(\theta^{\prime}\right)=\frac{\psi+\sqrt{\psi^{2}+4 a^{2} \sin ^{2} \theta^{\prime}}}{2 \sin \theta^{\prime}} .
$$




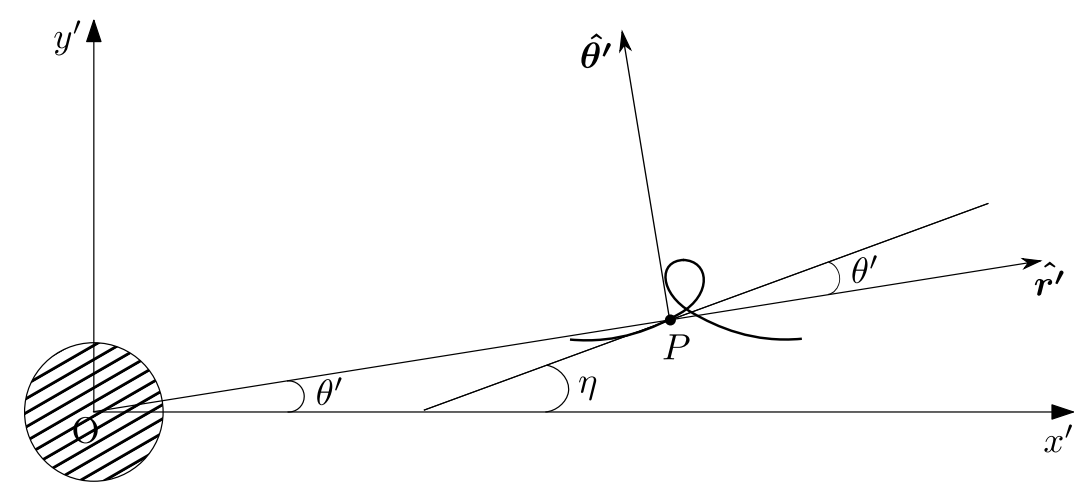

Figure 3.1: Schematic of the angles between the cylinder and the target particle $\left(\theta^{\prime}\right)$ as well as the angle that the tangent to the particle's curvature makes with the OX axis $(\eta)$.

Additionally, when expressed using the angle $\eta$ made by the tangent to the particle trajectory at a given point $P$ and the OX axis (see Figure 3.1, which shows the moment the cylinder is at the origin, $\mathrm{O}$ ) as the dependent variable and the arc-length $s$ along the particle trajectory as the independent variable, the equation describing the particle trajectories may be determined. In particular, rewriting the streamfunction in (3.14) in terms of $\sin ^{2} \theta^{\prime}$ and differentiating both sides with respect to $y$ (it should also be noted that $y$ and $y^{\prime}$ are equivalent under the transformation between the coordinate systems in Figure 1.1 so we may write $y^{\prime}=y$ ), we obtain

$$
\begin{aligned}
\sin ^{2} \theta^{\prime} & =\frac{y^{2}}{a^{2}}-\frac{\psi y}{a^{2}} \\
2 \sin \theta^{\prime} \cos \theta^{\prime} \frac{d \theta^{\prime}}{d y} & =\left(\frac{2 y}{a^{2}}-\frac{\psi}{a^{2}}\right) \\
\frac{d \theta^{\prime}}{d y} & =\frac{\left(\frac{2 y}{a^{2}}-\frac{\psi}{a^{2}}\right)}{\sin \left(2 \theta^{\prime}\right)} .
\end{aligned}
$$

According to Figure 3.1, we may write $\eta=2 \theta^{\prime}$. For an infinitesimal line element, 
$d s$, along a path taken by a particle we also have $d y / d s=\sin \left(2 \theta^{\prime}\right)$. Therefore,

$$
\frac{d \eta}{d s}=\frac{d\left(2 \theta^{\prime}\right)}{d y} \frac{d y}{d s}=2 \sin \left(2 \theta^{\prime}\right) \frac{d \theta^{\prime}}{d y}
$$

and thus, after substituting (3.16) the particle trajectories are of the form

$$
\frac{d \eta}{d s}=\frac{4}{a^{2}}\left(y-\frac{1}{2} \psi\right),
$$

implying that the trajectories are examples of "elasticas", a family of curves with a long history in mathematics [15]. The quantity $d \eta / d s$ represents the curvature of the trajectory and the connection with elasticas was first recognized by Milne-Thomson [11]. However, for our purposes, it is more convenient to start from equation (3.5) in which the independent variable is changed from $t$ to $\theta^{\prime}$ as shown in (3.2). More specifically, we have the following velocity components in our two coordinate systems

$$
\begin{aligned}
& u_{x}\left(r^{\prime}, \theta^{\prime}\right)=\frac{d x}{d t}=\frac{a^{2} \cos \left(2 \theta^{\prime}\right)}{r^{\prime 2}} \\
& u_{\theta}\left(r^{\prime}, \theta^{\prime}\right)=r^{\prime} \frac{d \theta^{\prime}}{d t}=\sin \theta^{\prime}\left(\frac{a^{2}}{r^{\prime 2}}+1\right) .
\end{aligned}
$$

which, when substituted into (3.3) (we will refer to the drift in the wakeless potential flow as $\xi_{1}$ ), yield

$$
\begin{aligned}
\xi_{1}\left(y_{0}\right) & =\int_{0}^{\pi} r^{\prime} \frac{u_{x}\left(r^{\prime}, \theta^{\prime}\right)}{u_{\theta}\left(r^{\prime}, \theta^{\prime}\right)} d \theta^{\prime} \\
& =\int_{0}^{\pi} \frac{a^{2} \cos \left(2 \theta^{\prime}\right)}{r^{\prime} \sin \theta^{\prime}\left(\frac{a^{2}}{r^{\prime 2}}+1\right)} d \theta^{\prime}
\end{aligned}
$$

for the drift. We may substitute for $a^{2} / r^{2}$ by solving (3.14). Furthermore, using 
relation (3.15) for $r^{\prime}$ and remembering that $\psi$ remains constant during a particle's motion, we may now obtain an expression for $\xi_{1}$ that depends solely on $\theta^{\prime}$ as the independent variable,

$$
\begin{aligned}
\xi_{1}\left(y_{0}\right) & =\int_{0}^{\pi} \frac{a^{2} \cos \left(2 \theta^{\prime}\right)}{r^{\prime} \sin \theta^{\prime}\left(2-\frac{\psi}{r^{\prime} \sin \theta^{\prime}}\right)} d \theta^{\prime} \\
& =\int_{0}^{\pi} \frac{a^{2} \cos \left(2 \theta^{\prime}\right)}{2 \sin \theta^{\prime}\left(\frac{\psi \pm \sqrt{\psi^{2}+4 a^{2} \sin ^{2} \theta^{\prime}}}{2 \sin \theta^{\prime}}\right)-\psi} d \theta^{\prime} \\
& =\int_{0}^{\pi} \frac{a^{2} \cos \left(2 \theta^{\prime}\right)}{ \pm \sqrt{\psi^{2}+4 a^{2} \sin ^{2} \theta^{\prime}}} d \theta^{\prime} .
\end{aligned}
$$

From now on, we will neglect the negative variant of the denominator because, due to symmetry, we can restrict our calculations to the upper half-plane only.

We will have to rely on some special functions to evalute the integral in (3.22). In particular, we will use the complete elliptic integrals of the first and second type

$$
\begin{aligned}
\mathcal{K} & =\int_{0}^{\pi / 2} \frac{1}{\sqrt{1-k^{2} \sin ^{2} \theta}}, d \theta \\
\mathcal{E} & =\int_{0}^{\pi / 2} \sqrt{1-k^{2} \sin ^{2} \theta} d \theta
\end{aligned}
$$

where the parameter $k \in \mathbb{R}$ is called the elliptic modulus. Starting with equation 
(3.22), we obtain

$$
\begin{aligned}
\xi_{1}\left(y_{0}\right) & =\int_{0}^{\pi} \frac{a^{2} \cos \left(2 \theta^{\prime}\right)}{\sqrt{\psi^{2}+4 a^{2} \sin ^{2} \theta^{\prime}}} d \theta^{\prime} \\
& =\int_{0}^{\pi} \frac{a^{2}\left(1-2 \sin ^{2} \theta^{\prime}\right)}{\sqrt{\psi^{2}+4 a^{2} \sin ^{2} \theta^{\prime}}} d \theta^{\prime} \\
& =\int_{0}^{\pi} \frac{a^{2}}{\sqrt{\psi^{2}+4 a^{2} \sin ^{2} \theta^{\prime}}} d \theta^{\prime}-\int_{0}^{\pi} \frac{2 a^{2} \sin ^{2} \theta^{\prime}}{\sqrt{\psi^{2}+4 a^{2} \sin ^{2} \theta^{\prime}}} d \theta^{\prime} \\
& =\mathcal{R}-\mathcal{S}
\end{aligned}
$$

where $\mathcal{R}$ and $\mathcal{S}$ refer to the two integrals in equation (3.24). We will first solve for $\mathcal{R}$ by making the substitution, $\theta=\theta^{\prime}-\frac{\pi}{2}$, such that $\sin \theta^{\prime}=\cos \theta$ and $\sin ^{2} \theta^{\prime}=1-\sin ^{2} \theta$. After some rearrangning, we get,

$$
\begin{aligned}
\mathcal{R} & =\int_{-\pi / 2}^{\pi / 2} \frac{a^{2}}{\sqrt{\psi^{2}+4 a^{2}\left(1-\sin ^{2} \theta\right)}} d \theta \\
& =\frac{a^{2}}{\sqrt{\psi^{2}+4 a^{2}}} \int_{-\pi / 2}^{\pi / 2} \frac{1}{\sqrt{1-\frac{4 a^{2}}{\psi^{2}+4 a^{2}} \sin ^{2} \theta}} d \theta .
\end{aligned}
$$

Next we can define the parameter in the elliptic function to be $k^{2}:=\frac{4 a^{2}}{\psi^{2}+4 a^{2}}$. Since the integrand is an even function, the integration can be reduced to the interval $[0, \pi / 2]$. Therefore, we obtain

$$
\mathcal{R}=\frac{1}{2} k^{2}\left(\frac{2 a}{k}\right) \int_{0}^{\pi / 2} \frac{1}{\sqrt{1-k^{2} \sin ^{2} \theta}} d \theta
$$

Which is exactly the complete elliptic integral of the first kind. Thus, we conclude that

$$
\mathcal{R}=\frac{1}{2} k^{2}\left(\frac{2 a}{k}\right) \mathcal{K}
$$


Next, to solve for $\mathcal{S}$, we follow the same approach,

$$
\begin{aligned}
\mathcal{S} & =4 a^{2} \int_{0}^{\pi / 2} \frac{\left(1-\sin ^{2} \theta\right)}{\sqrt{\psi^{2}+4 a^{2}\left(1-\sin ^{2} \theta\right)}} d \theta \\
& =k^{2}\left(\frac{2 a}{k}\right) \int_{0}^{\pi / 2} \frac{\left(1-\sin ^{2} \theta\right)}{\sqrt{1-k^{2} \sin ^{2} \theta}} d \theta \\
& =k^{2}\left(\frac{2 a}{k}\right)\left[\mathcal{K}-\int_{0}^{\pi / 2} \frac{\sin ^{2} \theta}{\sqrt{1-k^{2} \sin ^{2} \theta}} d \theta\right] \\
& =\left(\frac{2 a}{k}\right)\left[k^{2} \mathcal{K}-\int_{0}^{\pi / 2} \frac{k^{2} \sin ^{2} \theta}{1-k^{2} \sin ^{2} \theta} \sqrt{1-k^{2} \sin ^{2} \theta} d \theta\right] \\
& =\left(\frac{2 a}{k}\right)\left[k^{2} \mathcal{K}-\int_{0}^{\pi / 2}\left(\frac{1}{1-k^{2} \sin ^{2} \theta}-1\right) \sqrt{1-k^{2} \sin ^{2} \theta} d \theta\right] \\
& =\left(\frac{2 a}{k}\right)\left[k^{2} \mathcal{K}-\mathcal{K}+\mathcal{E}\right] .
\end{aligned}
$$

Thus, we have expressed $\mathcal{R}$ and $\mathcal{S}$ in equation (3.24) in terms of complete elliptic integrals of the first and second kind. Now putting the two together for the drift, we arrive at

$$
\xi_{1}\left(y_{0}\right)=\mathcal{R}-\mathcal{S}=\frac{2 a}{k}\left[\left(1-\frac{1}{2} k^{2}\right) \mathcal{K}-\mathcal{E}\right] .
$$

This is the equation which allows us to compute the individual particle drift in the wakeless potential flow. It relies on elliptic functions which cannot be written in terms of elementary functions, however, they may be evaluated using modern numerical tools. We note that the initial position of the particle is encoded in the value of the streamfunction $\psi$ appearing in the expression for $k$.

To extend the analysis using elliptic functions further, it is possible to find the particle's displacement $x$ as a function of the incomplete elliptic integral. The incomplete 
elliptic integrals of the first and second type may be written as

$$
\begin{aligned}
u & :=\int_{0}^{\theta^{\prime}-\pi / 2} \frac{1}{\sqrt{1-k^{2} \sin ^{2} \theta}} d \theta, \\
\mathcal{E}(u) & :=\int_{0}^{\theta^{\prime}-\pi / 2} \sqrt{1-k^{2} \sin ^{2} \theta} d \theta .
\end{aligned}
$$

where the parameter $k^{2}$ is chosen as before. Then $x(u)$ is given by

$$
x(u)=\frac{a}{k}\left[\left(1-\frac{1}{2} k^{2}\right) u-\mathcal{E}(u)\right] .
$$

We note that the drift corresponding to $t \in(-\infty, \infty)$, cf. (3.29), is then obtained by taking the limit $\theta^{\prime} \rightarrow \pi / 2$ in (3.30).

Likewise, it is possible to determine the particle's vertical position in terms of special functions. The Jacobi elliptic function may be defined as

$$
\operatorname{dn}(u):=\sqrt{1-k^{2} \sin ^{2}\left(\theta^{\prime}-\frac{\pi}{2}\right)} .
$$

Then, solving the streamfunction expression (3.14) for $y$ and substituting in $\operatorname{dn}(u)$,

$$
y(u)=\frac{a}{k}\left[\frac{\psi}{\sqrt{\psi^{2}+4 a^{2}}}+\operatorname{dn}(u)\right]=\frac{a}{k}\left[\frac{d k}{d \psi}+\operatorname{dn}(u)\right] .
$$

To conclude, in this section we have shown that with a careful analysis of the wakeless potential flow model, it is possible to obtain closed-form expressions for the resulting particle displacement as well as the drift in terms of elliptic functions. This will provide an important benchmark for which to compare our numerical results in Chapter 4.1. 
As regards the total drift area for the wakeless potential flow, using Darwin's theorem as in (3.9), the total drift area (which we will refer to as $D_{1}$ in the wakeless potential flow) can then be shown to be

$$
D_{1}=\pi
$$

These results will be illustrated in Chapter 4.2. 


\section{Chapter 4}

\section{Results}

In this Chapter we compare the trajectories of individual particles, their drift and the corresponding total drift areas in the three flows introduced in the previous Chapter. While, as reviewed in Chapter 3.3, these quantities can be determined analytically in the wakeless potential flow, they have to be computed numerically in the case of the Föppl and Kirchhoff flows, and the computational techniques are described and validated in Chapter 4.1. Finally, the main results are presented in Chapter 4.2.

\subsection{Numerical Computation of Particle Trajectories, Drift and Total Drift Area in the Föppl and Kirchhoff Flows}

Since explicit relations of the type (3.15) are not available for the Föppl and Kirchhoff flows, we need to resort to numerical computations in order to determine the particle trajectories, drift and the total drift area. The particle trajectories are computed as 
described in Chapter 3 by solving system (3.5) with the initial data $\mathbf{x}_{0}=\left[0, y_{0}\right]^{T}$, where $y_{0}>1$ is a parameter (we note that, when $y_{0}=1 \mathrm{in} \mathrm{Föppl} \mathrm{flow,} \mathrm{the} \mathrm{particle}$ is on the streamline connected to the stagnation point and the drift $\xi(1)$ is infinite). In the case of the Föppl flow the particle trajectories are additionally parameterized by the vortex circulation $\Gamma$. The velocity on the right-hand side of (3.5) is obtained, respectively, by complex-differentiating potential (2.2) and using expressions (2.12) in the two cases. System (3.5) is integrated for different values of $y_{0}$ and, in the case of the Föppl flow, $\Gamma$ using MATLAB routines ode23 and ode45 with adaptive adjustment of the time step. Numerical evaluation of the drift, given by an improper integral (3.1), is a subtle issue requiring judicious choice of the truncation $[-T, T]$ of the original unbounded interval $(-\infty, \infty)$. Questions concerning the partial drift, evaluated for finite times $t \in[-T, T]$, were investigated in $[21,25]$ where it was shown that such truncation of the integration domain leads to nontrivial corrections to the drift as defined in (3.1). In order to exclude these finite-time effects from the numerical integration, one has to make sure that $T$ is chosen sufficiently large. For Föppl flow, this is achieved by setting $T$ close to realmax, the largest positive floating-point number in the IEEE double-precision standard [40], which is of the order $\mathcal{O}\left(10^{300}\right)$ and then balancing the accuracy with the computational time by adjusting the relative and absolute tolerances, RelTol and AbsTol, in the routines ode23 and ode45. The drift actually converges to a number which is within a very small tolerance of the exact result well before $t$ reaches $T=\mathcal{O}\left(10^{300}\right)$, however, we select large values of $T$ to ensure that we achieve the largest accuracy possible (which may not be necessarily required to obtain satisfactory estimates in many applications). Owing to the adaptive adjustment of the time step employed in these 
routines, the total computational time required for a single particle trajectory does not typically exceed one minute on a state-of-the-art workstation even for the finest tolerances. This approach is validated by computing the particle trajectories $\mathbf{x}\left(t ; y_{0}\right)$ and the associated drift $\xi\left(y_{0}\right)$ numerically for the wakeless potential flow (obtained setting $\Gamma=0$ in (2.2)) and then comparing them to the analytical expressions (3.29), (3.31) and (3.33) (since these formulas involve special functions, care must be taken to enforce a required level of precision in the evaluation of these functions as well). The results obtained for a single trajectory with $y_{0}=2$ are presented in Figure 4.1a, where we show a segment of the particle trajectory computed numerically and given by expressions (3.31) and (3.33), and in Figure 4.1b in which we show the difference between the exact drift value $\xi_{1}(2)=2.011398641052742 \times 10^{-1}$ and its numerical approximation $\hat{\xi}_{1}(2)$ for different fixed RelTol and varying AbsTol. As is evident from Figure 4.1b, the error in the evaluation of the drift is rather small and decreases algebraically with the refinement of both RelTol and AbsTol, Thus, in all subsequent calculations we will use routine ode45 with RelTol $=$ AbsTol $=10^{-13}$.

Unlike in the case of the wakeless and Föppl flow where $T$ was allowed to extend close to realmax, in Kirchhoff flow we have to restrict the truncation of the time axis to $T=10^{3}$ which is due to the failure of Newton's method applied to (2.13) to converge for such large values of $t$. However, since the amount of data obtained despite such a truncation in time is sufficient and since the structure of the flow advecting the particles does not change much when $|t|>T$, we will compensate for this by extrapolating the velocity for large times. Since, as will be shown below, the velocity field following the particle trajectory is for sufficiently large $t$ a power-law 


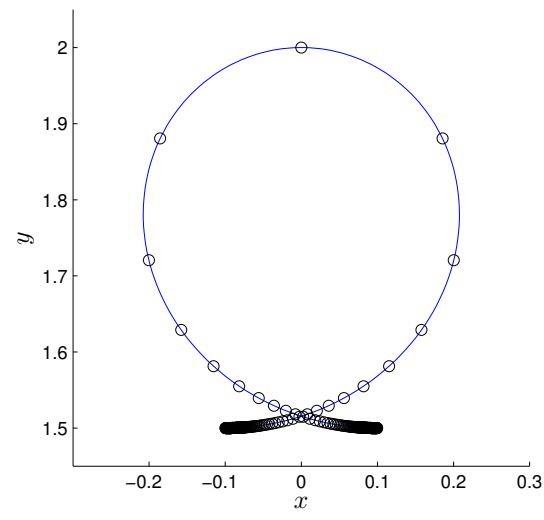

(a)

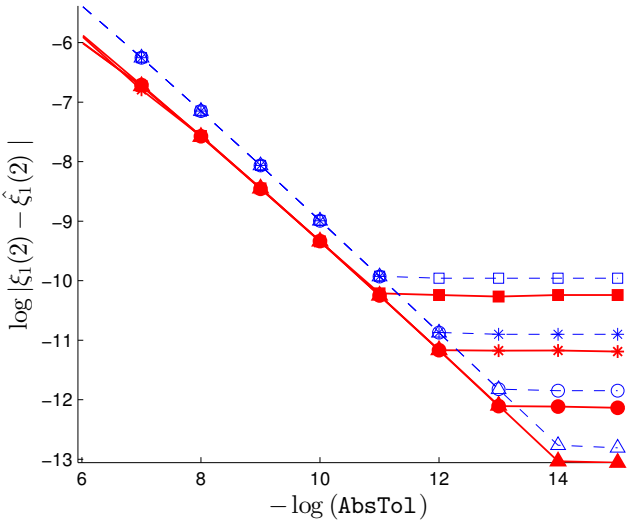

(b)

Figure 4.1: (a) Particle trajectories corresponding to $y_{0}=2$ in the wakeless potential flow obtained numerically with RelTol $=\mathrm{AbsTol}=10^{-13}$ (symbols) and evaluated analytically using formulas (3.31) and (3.33) (solid line); (b) error between the corresponding drift $\xi_{1}(2)$, cf. (3.29), and its numerical approximation $\hat{\xi}_{1}(2)$ computed using routines ode23 (open symbols) and ode45 (filled symbols) for RelTol $=10^{-10}$ (squares), $10^{-11}$ (stars), 10 $0^{-12}$ (circles), and $10^{-13}$ (triangles). In this example, it takes $t \approx \mathcal{O}\left(10^{16}\right)$ time units for the particle to converge to machine precision.

function of time, this extrapolation will be performed using the formula

$$
h(t)=c t^{\beta}
$$

where $c \in \mathbb{R}$ and $\beta<0$, using 10 data points corresponding to the largest available times.

As regards evaluation of the total drift area $D$, three different approaches are used: definition formula (3.4), or more conveniently (3.8), added-mass formula (3.9) and Taylor's theorem (3.12)-(3.13). In the first approach the parameter space $y_{0}$ is discretized in such a way that the relative variation of $\xi\left(y_{0}\right)$ between two adjacent discrete values of $y_{0}$ would not exceed $1 \%$. The function $g\left(y_{0}\right)$ and its derivative 
needed in (3.8) are identified for the Föppl flow as follows

$$
\begin{gathered}
g\left(y_{0}\right)=-\left(y_{0}-\frac{1}{y_{0}}\right)+\frac{\Gamma}{2 \pi}\left[\log \left(\frac{\sqrt{x_{1}^{2}+\left(y_{0}+y_{1}\right)^{2}}}{\sqrt{x_{1}^{2}+\left(y_{0}-y_{1}\right)^{2}}}\right)\right. \\
+\log \left(\frac{\left.\sqrt{\left(\frac{x_{1}}{x_{1}^{2}+y_{1}^{2}}\right)^{2}+\left(y_{0}-\frac{y_{1}}{x_{1}^{2}+y_{1}^{2}}\right)^{2}}\right]}{\left.\left.\sqrt{\left(\frac{x_{1}}{x_{1}^{2}+y_{1}^{2}}\right)^{2}+\left(y_{0}+\frac{y_{1}}{x_{1}^{2}+y_{1}^{2}}\right)^{2}}\right)\right]}\right. \\
g^{\prime}\left(y_{0}\right)=-\left(1+\frac{1}{y_{0}^{2}}\right)+\frac{\Gamma}{2 \pi}\left[\frac{y_{0}+y_{1}}{x_{1}^{2}+\left(y_{0}+y_{1}\right)^{2}}-\frac{y_{0}-y_{1}}{x_{1}^{2}+\left(y_{0}-y_{1}\right)^{2}}\right. \\
\left.+\frac{y_{0}-\frac{y_{1}}{x_{1}^{2}+y_{1}^{2}}}{\left(\frac{x_{1}}{x_{1}^{2}+y_{1}^{2}}\right)^{2}+\left(y_{0}-\frac{y_{1}}{x_{1}^{2}+y_{1}^{2}}\right)^{2}}-\frac{y_{0}+\frac{y_{1}}{x_{1}^{2}+y_{1}^{2}}}{\left(\frac{x_{1}}{x_{1}^{2}+y_{1}^{2}}\right)^{2}+\left(y_{0}+\frac{y_{1}}{x_{1}^{2}+y_{1}^{2}}\right)^{2}}\right] .
\end{gathered}
$$

Concerning the computation of the total drift area via Taylor's theorem (3.12)-(3.13), the two Föppl vortices and their images inside the cylinder make the contributions $m= \pm \frac{\Gamma}{2 \pi i}$ each, whereas the dipole at the origin representing the cylinder with unit radius contributes $\mu=1$. Therefore, equation (3.12) becomes

$$
\begin{aligned}
M & =2 \pi \Re\left(\frac{\Gamma}{2 \pi i} z_{1}-\frac{\Gamma}{2 \pi i \overline{z_{1}}}-\frac{\Gamma}{2 \pi i} z_{2}+\frac{\Gamma}{2 \pi i \overline{z_{2}}}+1\right)-B \\
& =2 \pi+2 \Gamma\left(y_{1}-\frac{y_{1}}{x_{1}^{2}+y_{1}^{2}}\right)-B .
\end{aligned}
$$

Since it does not appear possible to find an analytic expression for $B$ representing the area of the recirculation bubble, it has to be evaluated numerically using (3.13). 


\subsection{Comparison of Particle Trajectories, Drift and Total Drift Area in Flows with Different Wake Models}

The particle trajectories corresponding to several different initial positions $\mathbf{x}_{0}=$ $\left[0, y_{0}\right]^{T}$ are shown in Figure 4.2 for the wakeless potential flow, the Föppl flow with different circulations $\Gamma$ and for the Kirchhoff flow. The initial positions corresponding to the indicated cylinder locations are marked with circles, whereas crosses indicate the particle positions at the same instances of time in the different cases. First, we see that in the wakeless potential flow (Figure 4.2a) all the particle trajectories have the form of elastica curves symmetric with respect to the OY axis. The presence of the vortex wakes in the Föppl flows breaks the fore-and-aft symmetry of the trajectories and, for small values of $y_{0}$, spawns a second loop on the trajectory which becomes larger for increasing vortex circulations $\Gamma$. The presence of this secondary loop results from the fact that, for sufficiently small $y_{0}$, the transverse component $u_{y}$ of the particle velocity must change sign when the particle is flowing around the recirculation region. The total drift areas produced by the fluid displacements shown in Figures 4.2(a) and (c) are approximately equal, cf. (3.8), even though the individual particles with the same initial locations have quite different trajectories.

Next, in Figure 4.3a, we show the drift $\xi$ of the individual particles as a function of the circulation $\Gamma$ and, in Figure 4.3b, as a function of the initial distance $y_{0}$ form the horizontal axis. Since this is how it is often presented, the latter data is replotted in Figure 4.3c using the linear scaling with the drift $\xi$ marked on the horizontal axis and the vertical axis representing $y_{\infty}$, cf. (3.6). In Figure 4.3a we see that the 


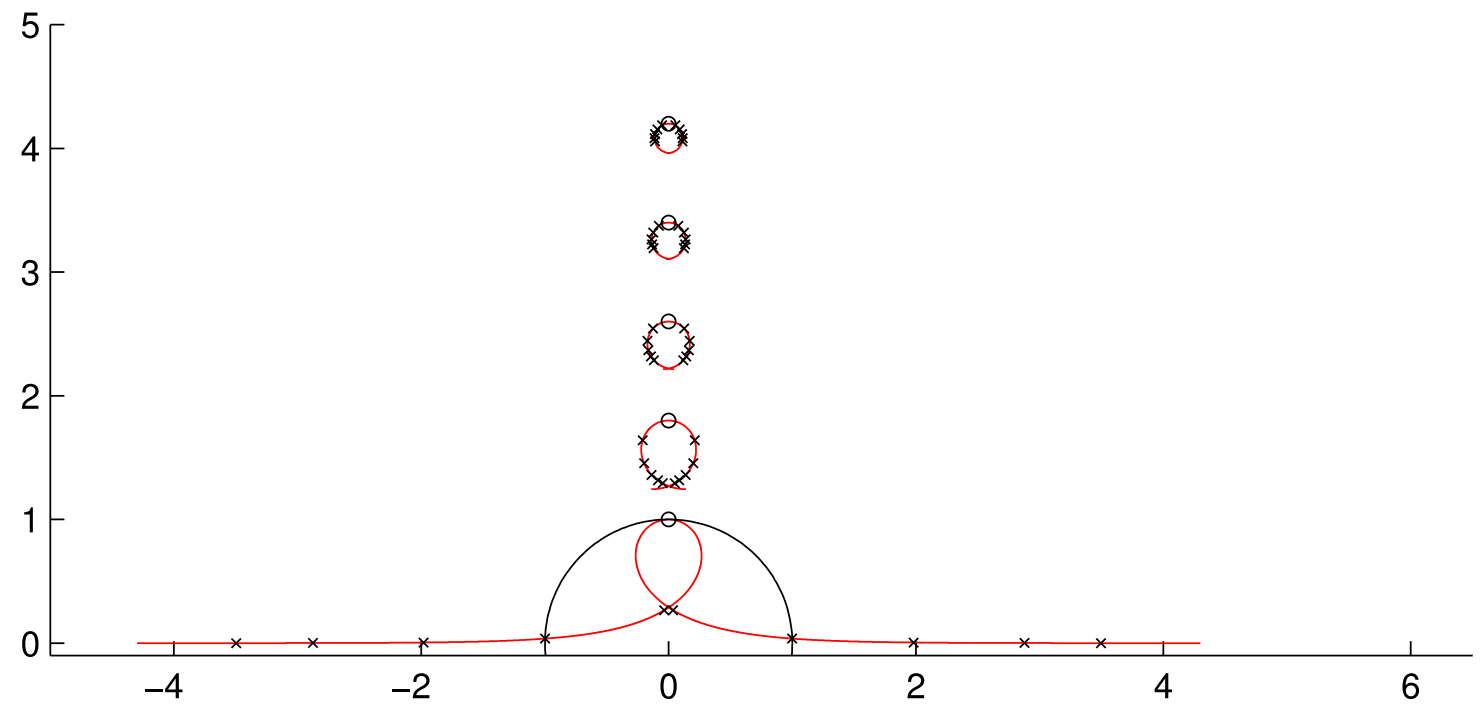

(a) wakeless potential flow $(\Gamma=0)$

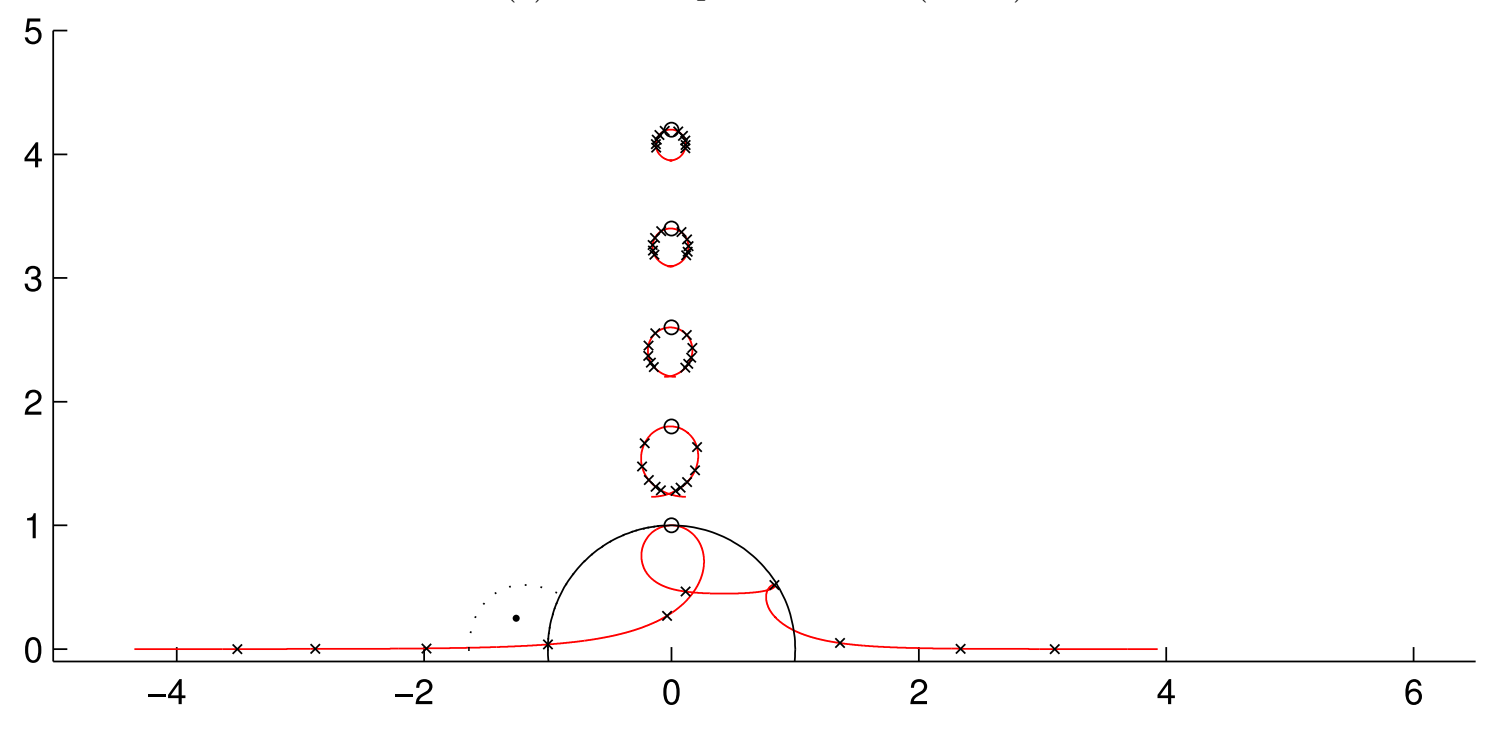

(b) $\Gamma=1.9663$

Figure 4.2: Particle trajectories for different initial conditions $\mathbf{x}_{0}=\left[0, y_{0}\right]^{T}$ in the wakeless potential flow (a), the Föppl flow with different circulations (b,c,d) and the Kirchhoff flow (e). The x's represent the particle positions at unit time intervals, whereas the o's correspond to the particle positions at $t=0$, at which the cylinder, recirculation bubble for Föppl flow and the cavity for Kirchhoff flow are also indicated. 


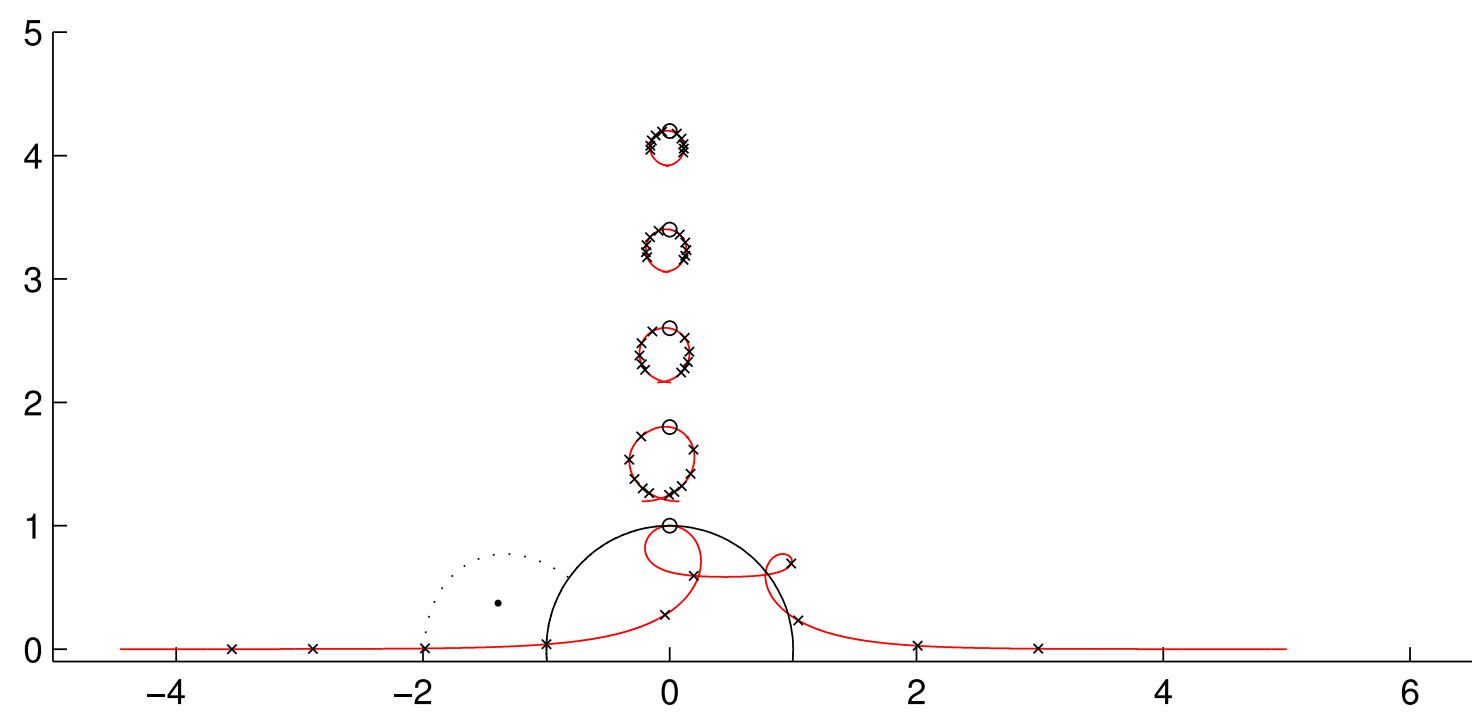

(c) $\Gamma=3.595$

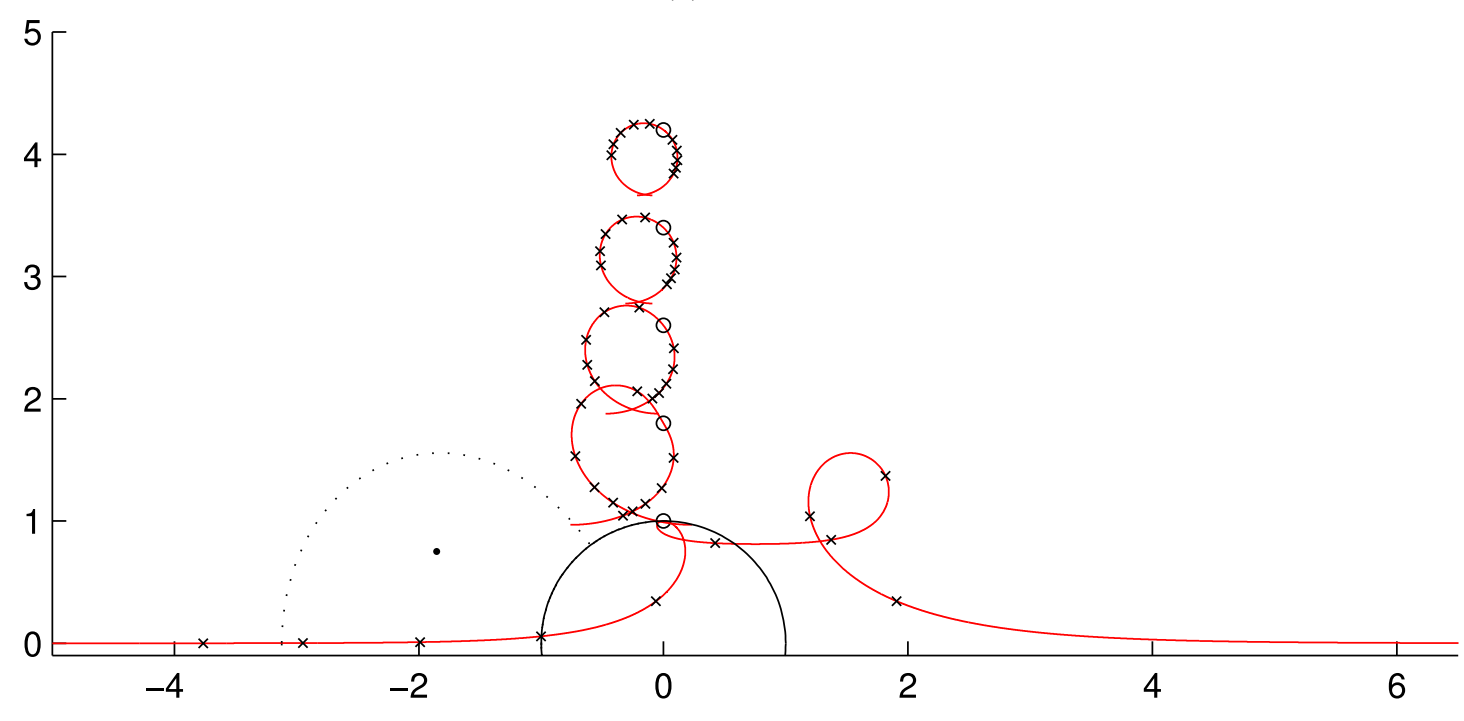

(d) $\Gamma=8.8357$

Figure 4.2: (Continued, see previous caption for details) 


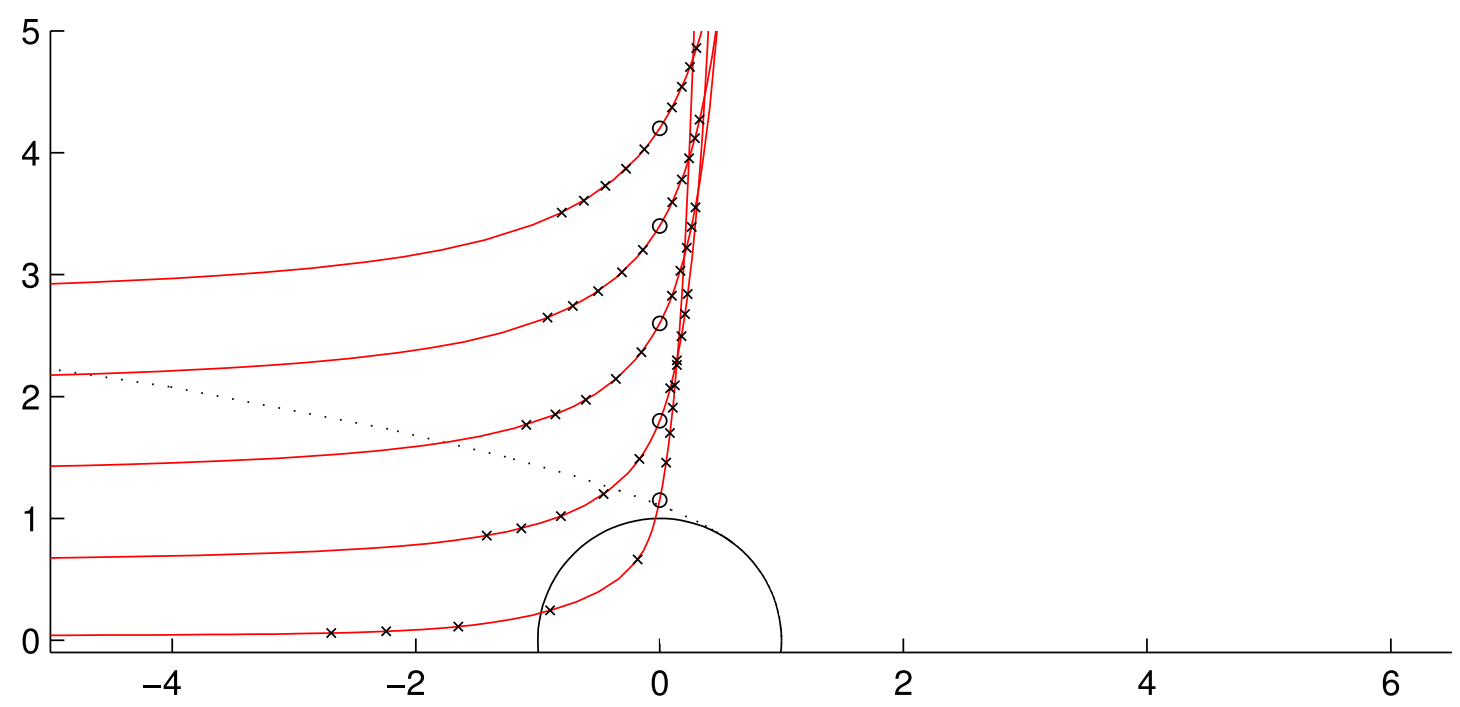

(e) Kirchhoff flow

Figure 4.2: (Continued, see previous caption for details)

dependence of the drift $\xi$ on the vortex circulation $\Gamma$ is not monotonous regardless of the initial position of the particle. Moreover, in a certain range of $\Gamma$ there are two initial positions $y_{0}$ such that the corresponding drift $\xi\left(y_{0}\right)$ is equal to the drift in the wakeless flow. While for sufficiently large circulations the drift ultimately increases as compared to the wakeless flow (corresponding to $\Gamma=0$ ), for small values of $\Gamma$ the drift is actually reduced. In other words, for every $y_{0}>1$ there exists a particular circulation $\Gamma_{0}>0$ such that the Föppl flow has the same drift $\xi$ as the wakeless flow. This circulation is a nonmonotonous function of the distance $y_{0}$ from the flow centerline. In addition to confirming these observations, Figure 4.3b shows that drift $\xi\left(y_{0}\right)$ is a decreasing function of $y_{0}$ which exhibits two distinct asymptotic regimes (see Chapter 5 for more details on this).

It turns out that, regardless of the initial position $\mathbf{x}_{0}=\left[0, y_{0}\right]^{T}$, in the Kirchhoff flow drift (3.1) is unbounded. This is evident from Figure 4.4 showing an extrapolation 
using formula (4.1) of the velocity component $u_{x}(t)$ following the particle trajectory for large positive and negative times. We observe that, while for positive times the asymptotic behavior is characterized by the exponent $\beta=-1.1172$, for negative times the exponent is $\beta=-0.5092$ implying that $u_{x}(t)$ is not in fact integrable. Although for brevity in Figure 4.4 the data was shown for one trajectory only (corresponding to $\left.y_{0}=5\right)$ analogous results we also obtained for other trajectories. Thus, the drift data is not shown for the Kirchhoff flow in Figure 4.3.

Finally, in Figure 4.5, we show the dependence of the total drift area $D$ on the vortex circulation $\Gamma$ computed using the three methods discussed in Chapter 4.1, all of which show excellent agreement. We see that the total drift area exhibits a welldefined minimum which is a manifestation of the competing effects observed in Figure 4.3a. The smallest drift area $D=2.93$ is achieved for $\Gamma=1.97$, whereas for $\Gamma=3.6$ drift area is approximately $D=\pi$, the same as in the wakeless potential flow. The particle trajectories corresponding to these two cases are shown in Figures $4.2 \mathrm{~b}$ and $4.2 \mathrm{c}$. 


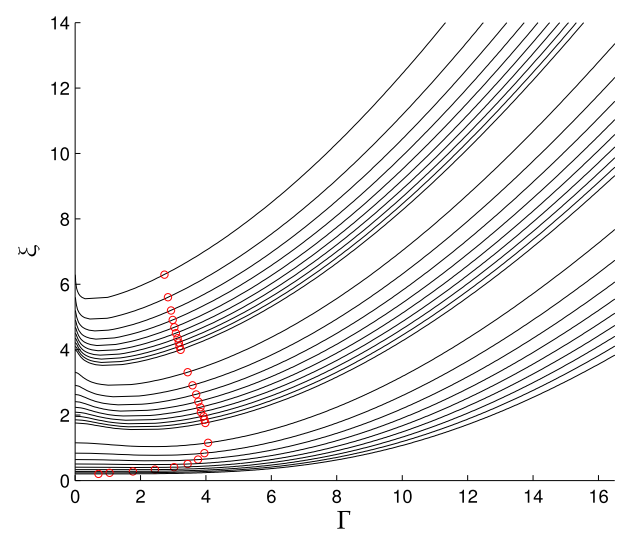

(a)

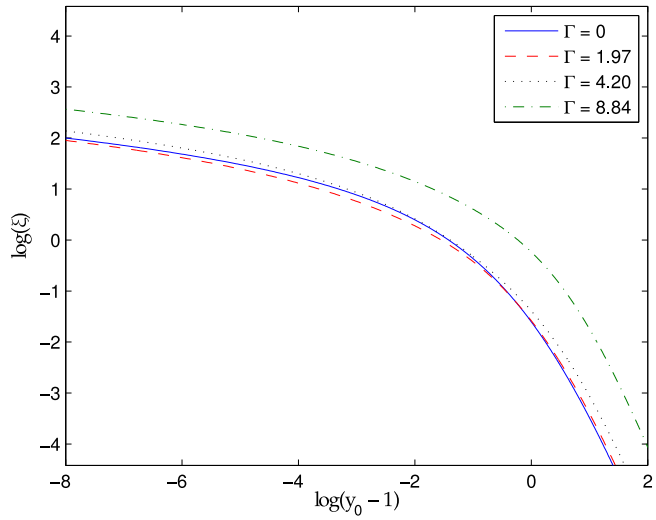

(b)

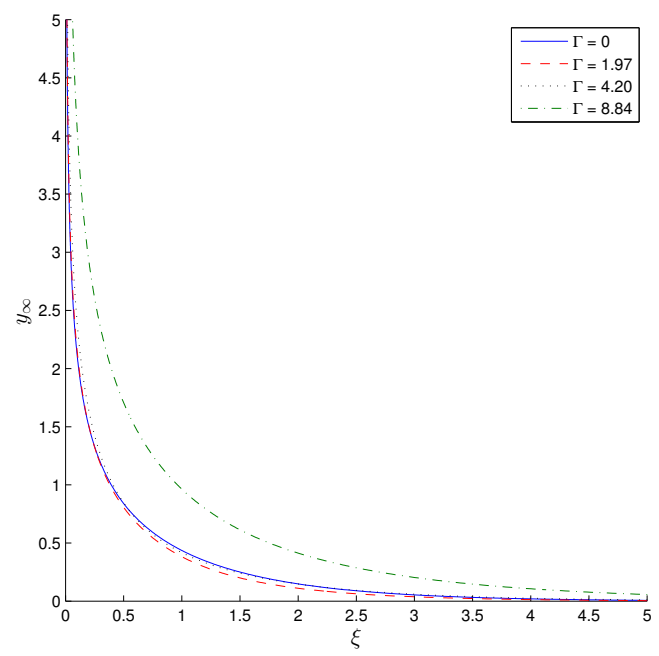

(c)

Figure 4.3: Dependence of drift $\xi$ on (a) the vortex circulation $\Gamma$ for initial particle positions $y_{0} \in\{1.001,1.002, \ldots, 1.01,1.02, \ldots, 1.1,1.2, \ldots, 2.0\}$ (larger $y_{0}$ corresponding to lower curves), (b) the initial distance $y_{0}$ and (c) the distance $y_{\infty}$ from the flow centerline measured at infinity, cf. (3.6), for the circulation values indicated in the legend. 


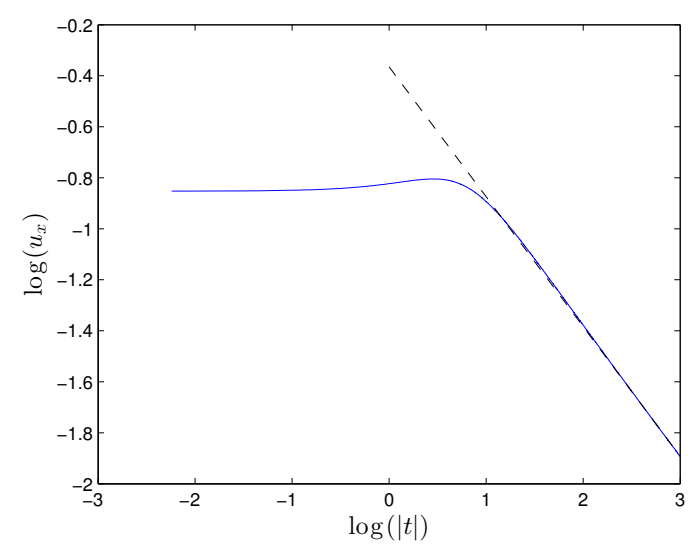

(a) $t<0, \beta=-0.5092$.

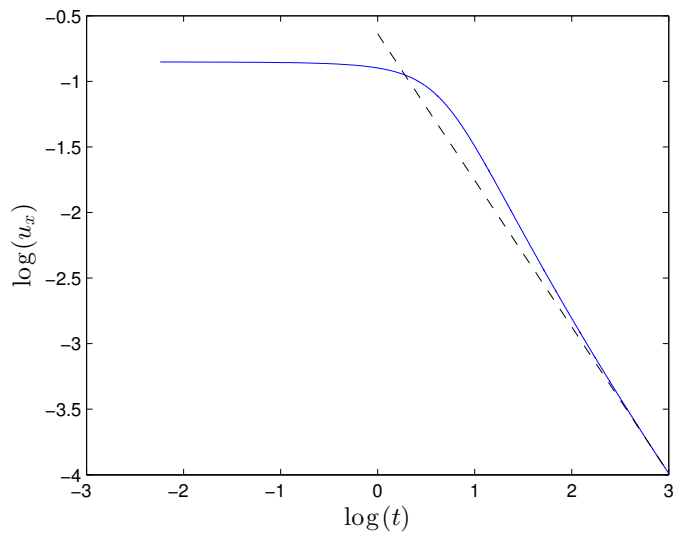

(b) $t>0, \beta=-1.1172$.

Figure 4.4: Behavior of the velocity component $u_{x}$ following the trajectory of the particle located at $y_{0}=5$ at $t=0$ in Kirchhoff flow for large (a) negative and (b) positive times (solid line); the dashed line represents the power-law fit (4.1) with the exponent values indicated in the captions.

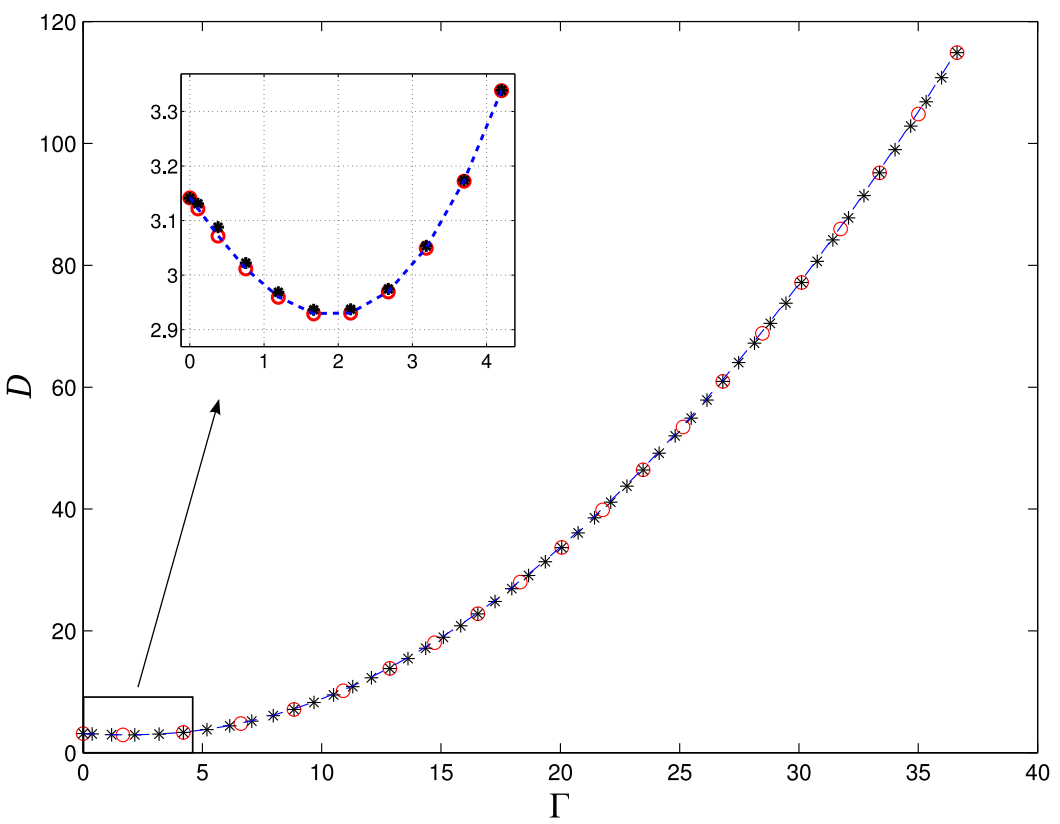

Figure 4.5: Total drift area $D$ in the Föppl flows as a function of the vortex circulation $\Gamma$ evaluated based on definition formula (3.8) (empty circles), added-mass formula (3.9) (crosses) and Taylor's theorem (3.12)-(3.13) (dashed line). 


\subsection{Discussion}

The particle trajectories in Föppl and Kirchhoff flows are quite different (cf. Figures $4.2 \mathrm{~b}-\mathrm{d}$ and $4.2 \mathrm{e}$ ). In Föppl flow for certain values of $\Gamma$ and $y_{0}$ the particle trajectories exhibit a secondary loop corresponding to the instant of time when the particle change direction to circumnavigate the recirculation bubble. An interesting, and perhaps somewhat unexpected, finding is that while for large values of circulation $\Gamma$ the presence of the recirculation region in Föppl flow increases the total drift area, an opposite effect occurs for smaller values of $\Gamma$ (Figure 4.3a). The increase of the total drift area for large $\Gamma$ can be understood by analyzing the particle trajectories in the context of changes to the flow topology. Inspection of Figure 4.2a, corresponding to the wakeless potential flow, reveals that the largest displacement occurs when the particle is close to one of the stagnation points (front or rear). The presence of the wake vortices in Föppl flow introduces another stagnation point (see Figure 2.1c) in the neighborhood of which particles can be trapped and dragged for a long time. This effect is illustrated in Figure 4.6 where we show several particle trajectories in the neighborhood of the separation point where the boundary of the recirculation zone meets the obstacle. Symbols on the trajectories mark positions at equal time intervals, indicating that the particles closer to the separation point are trapped there for a longer time. Passage near this separation point corresponds to the dragging of the particle before the second loop in the trajectories shown in Figures 4.2b-d. There are some interesting similarities and differences with respect to the wake effects on the drift in Stokes flows reported in [8]. In both cases the drift of an individual par-

ticle decays as $y_{0}^{-3}$ when the particle's position becomes large, cf. (5.2). This is a consequence of the fact that both the Föppl flow considered here and the Stokesian 
swimmer flow studied in [8] have a dipolar far-field representation (even though the spatial dimensions are different). On the other hand, in contrast to the behavior observed here, in the Stokes case a significant reflux (negative particle displacements) was observed resulting in a negative total drift volume corresponding to large wake sizes. This negative displacement which was observed for particles far from the obstacle shows that there are some differences in the far-field representation of velocity in 3D Stokesian and 2D inviscid flows that affects drift. More specifically, in the Stokesian case, the sign of the far-field depends on the distinction whether the swimmer (i.e. obstacle) is a pusher or a puller. However, when computing the drift assuming the far-field expansion of the velocity, this distinction only affects the less significant terms, and the net result is that the drift is negative.

In regard to Kirchhoff flow we demonstrated that drift $\xi$ of individual particles is in fact not bounded and, consequently, the total drift area is not defined either. This finding should not be surprising, given that Kirchhoff flow has an infinite open wake (and hence can be "seen" by the particles as a moving body of an infinite extent). We note that another instance in which an unbounded total drift volume was found was the Stokes flow past a spherical droplet [41]. Since like Kirchhoff flow and in contrast to the Stokesian swimmers analyzed in [8], this flow is characterized by a finite drag, we may by analogy conjecture that unbounded total drift area is a feature of steady flows in unbounded domains which exhibit a nonzero drag. 


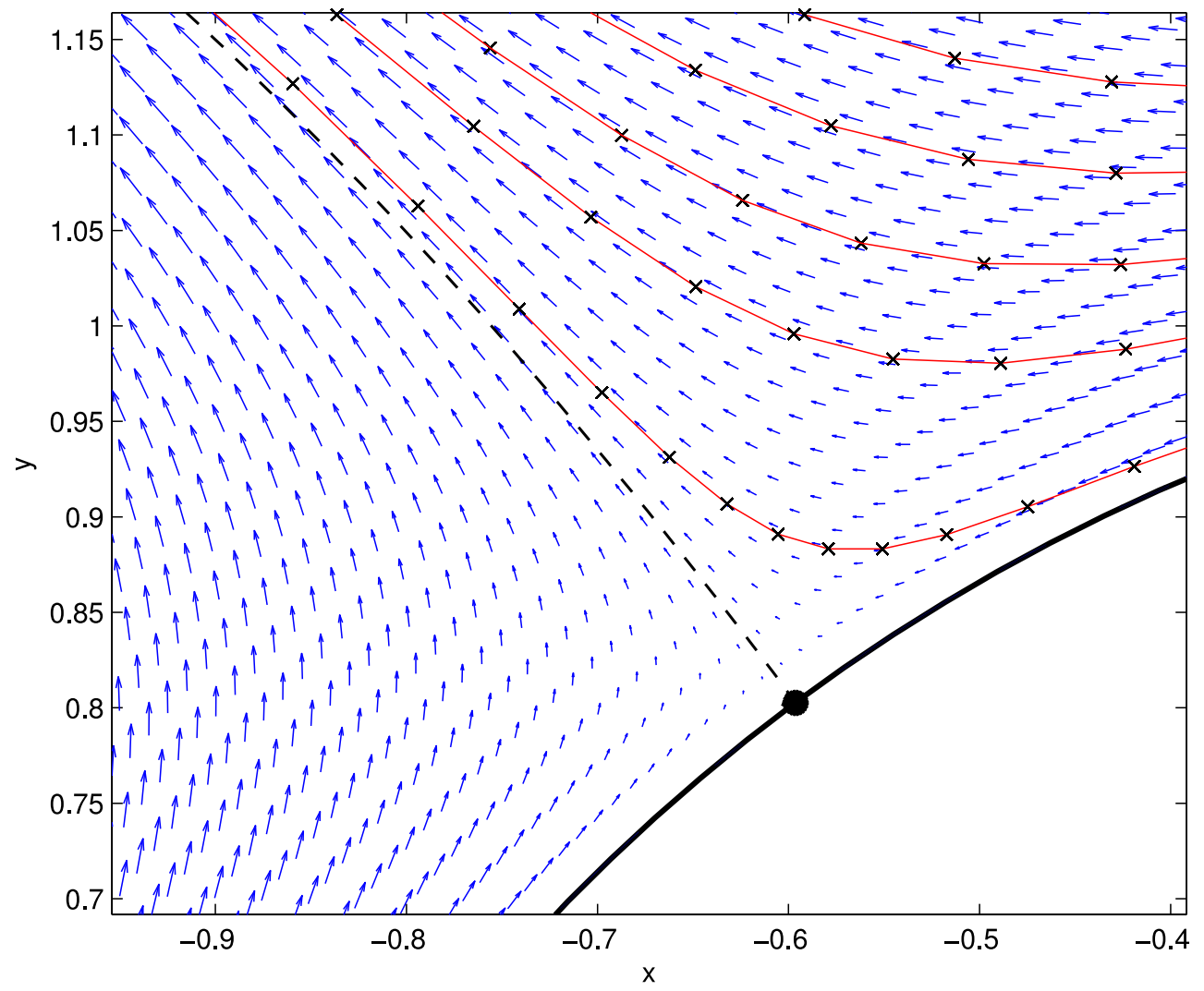

Figure 4.6: Neighborhood of the stagnation point (marked with a large dot) where the recirculation zone separatrix (dashed line) separates from the obstacle boundary (thick solid line). Particle trajectories are shown with thin solid lines with markers indicating positions at equal time intervals. 


\section{Chapter 5}

\section{Asymptotic Analysis}

In this section we derive expressions characterizing the drift when the parameters take some limiting values. The asymptotic study of the drift in the wakeless potential flow as $y_{0} \rightarrow 1$ and $y_{0} \rightarrow \infty$ is presented in [1], and our approach will build on this analysis.

As was discussed in Section 4.2, the drift of a particle in Föppl flow depends on two parameters, namely, the vortex circulation $\Gamma$ and the initial distance $y_{0}$ between the particle and the flow centerline.

A first, trivial, observation is that in the limit $\Gamma \rightarrow 0$ the drift of the wakeless potential flow is obtained uniformly in $y_{0}$. Here we will consider the limit $y_{0} \rightarrow$ $\infty$. Since the required transformations are rather complicated, requiring the use of symbolic algebra tools (Maple), for brevity below we will only highlight the key steps.

We start by taking the Taylor expansion of the velocity component $u_{x}$ in equation (3.5) about the initial position of the particle $\mathbf{x}_{0}=\left[0, y_{0}\right]^{T}$ and truncate it at the order $\mathcal{O}\left(\left\|\mathbf{x}-\mathbf{x}_{0}\right\|^{2}\right)$. This is justified by the observation, cf. Figure 4.2, that for large $y_{0}$ the particle trajectories are close to being circular and in the proximity of $\mathbf{x}_{0}$ 
Our goal will be to integrate this expansion with respect to time, cf. (3.1), but first we have to substitute for $x(t)$ and $y(t)$ to make the expansion a function of $t$ only. In the limit $y_{0} \rightarrow \infty$ the trajectories $\mathbf{x}(t)$ can be approximated with the solutions $\tilde{\mathbf{x}}(t)=[\tilde{x}(t), \tilde{y}(t)]^{T}$ of system (3.5) in which the right-hand side is evaluated at $\mathbf{x}_{0}$, i.e., $d \tilde{\mathbf{x}}(t) / d t=\mathbf{u}\left(\left[0, y_{0}\right]^{T}, t\right)$, which is written out as

$$
\begin{aligned}
\frac{d \tilde{x}}{d t}= & \frac{t^{2}-y_{0}^{2}}{\left(t^{2}+y_{0}^{2}\right)^{2}}+\frac{\Gamma}{2 \pi}\left[-\frac{y_{0}-y_{1}}{\left(-t-x_{1}\right)^{2}+\left(y_{0}-y_{1}\right)^{2}}+\frac{y_{0}-\frac{y_{1}}{x_{1}^{2}+y_{1}^{2}}}{\left(-t-\frac{x_{1}}{x_{1}^{2}+y_{1}^{2}}\right)^{2}+\left(y_{0}-\frac{y_{1}}{x_{1}^{2}+y_{1}^{2}}\right)^{2}}\right. \\
& \left.+\frac{y_{0}+y_{1}}{\left(-t-x_{1}\right)^{2}+\left(y_{0}+y_{1}\right)^{2}}-\frac{y_{0}+\frac{y_{1}}{x_{1}^{2}+y_{1}^{2}}}{\left(-t \frac{x_{1}}{x_{1}^{2}+y_{1}^{2}}\right)^{2}+\left(y_{0}+\frac{y_{1}}{x_{1}^{2}+y_{1}^{2}}\right)^{2}}\right] \\
\frac{d \tilde{y}}{d t}= & -\frac{2 t y_{0}}{\left(t^{2}+y_{0}^{2}\right)^{2}}+\frac{\Gamma}{2 \pi}\left[\frac{-t-x_{1}}{\left(-t-x_{1}\right)^{2}+\left(y_{0}-y_{1}\right)^{2}}-\frac{x_{1}}{\left(-t-\frac{x_{1}}{x_{1}^{2}+y_{1}^{2}}\right)^{2}+\left(y_{0}-\frac{y_{1}}{x_{1}^{2}+y_{1}^{2}}\right)^{2}}\right. \\
& \left.-\frac{-t-x_{1}}{\left(-t-x_{1}\right)^{2}+\left(y_{0}+y_{1}\right)^{2}}+\frac{-t-\frac{x_{1}}{x_{1}^{2}+y_{1}^{2}}}{\left(-t-\frac{x_{1}}{x_{1}^{2}+y_{1}^{2}}\right)^{2}+\left(y_{0}+\frac{y_{1}}{x_{1}^{2}+y_{1}^{2}}\right)^{2}}\right] .
\end{aligned}
$$

Relations (5.1a)-(5.1b) are integrated analytically for $\tilde{x}(t)$ and $\tilde{y}(t)$ and, before the resulting expressions are substituted in the series expansion of $u_{x}$, they are expanded in a Taylor series with respect to $\Gamma$ which is assumed small. Noting (2.4) and relations $y_{1}=\left(r_{1}^{2}-1\right) /\left(2 r_{1}\right)$ and $r_{1}=\sqrt{2 \sqrt{x_{1}^{4}-x_{1}^{2}+1}+2 x_{1}^{2}-1} / \sqrt{3}$, this expansion can be re-expressed only in terms of $x_{1}$, which is the downstream coordinate of the Föppl vortex. Finally, integrating the resulting expression from $t=-\infty$ to $t=\infty$ and keeping only the leading-order term in $y_{0}$, we obtain the following approximation to the drift

$$
\xi=\frac{\pi}{2 y_{0}^{3}}\left[1+64\left(x_{1}+1\right)^{4}+192\left(\left(x_{1}+1\right)^{5}+\left(x_{1}+1\right)^{6}\right)\right]+\mathcal{O}\left(\left(x_{1}+1\right)^{7}\right)
$$

valid for $y_{0} \rightarrow \infty$ and $x_{1} \rightarrow-1$ (equivalently, $\Gamma \rightarrow 0$ ). As is evident from this 


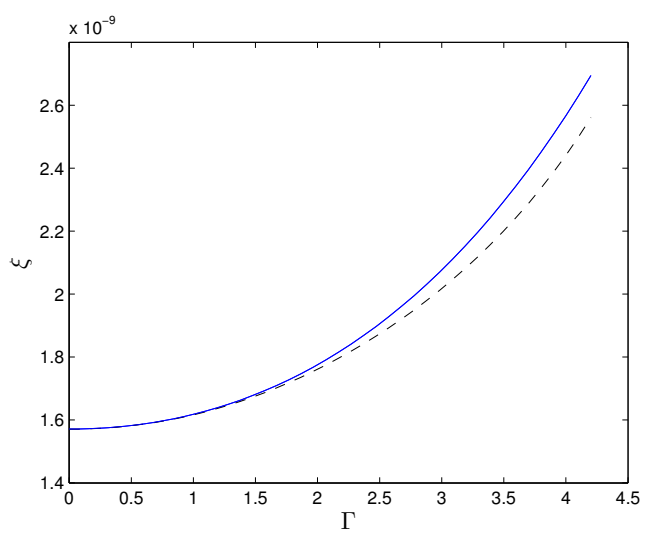

(a)

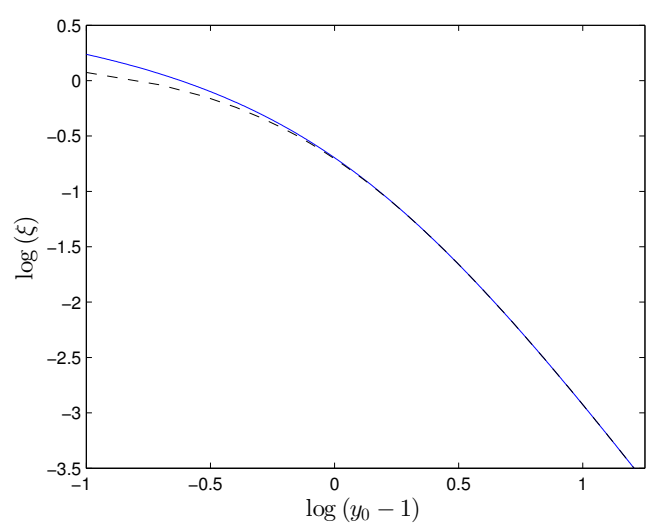

(b)

Figure 5.1: Dependence of drift $\xi$ on (a) the circulation $\Gamma$ for $y_{0}=1000$ and (b) the initial particle position $y_{0}$ for $\Gamma=0.38023$; solid lines represent the actual data whereas the dashed lines correspond to asymptotic formula (5.2).

relation, the presence of the Föppl vortices introduces a correction to the expression $\pi /\left(2 y_{0}^{3}\right)$ characterizing the drift in the wakeless potential flow in the limit $y_{0} \rightarrow \infty$ [1]. Asymptotic relation (5.2) is compared to the actual data for $\Gamma \rightarrow 0$ in Figure 5.1a and for $y_{0} \rightarrow \infty$ in Figure 5.1b showing a very good agreement in both cases. Analysis of the drift in the presence of the Föppl vortices in the limit $y_{0} \rightarrow 0$ is more complicated and is beyond the scope of the present study. 


\section{Chapter 6}

\section{Conclusions and Outlook}

In this study we presented a comprehensive analysis, based on careful numerical computations supported in some regimes by asymptotic analysis, of the effects of vortex wakes on the Darwinian drift induced by steadily translating obstacles. We focused on the Föppl and Kirchhoff flows featuring, respectively, a closed and open wake, which were compared to the wakeless potential flow used as a reference. We also discussed three different approaches to the computation of the total drift area, with the method based on Taylor's theorem leading to a decomposition of $D$ into a "universal" part and a "flow-specific" part, in analogy with the decomposition established in [8] for the Stokes flow.

We demonstrated that in the case of Föppl flow, particles close to the obstacle follow trajectories featuring a second loop. In addition, these findings show that in general, the total drift area increases with the size of the wake, except for a particular interval when $\Gamma$ is small, for which the drift area decreases when compared with the wakeless case. Meanwhile, by extrapolating the numerical data for horizontal velocity, we have shown that drift in the case of Kirchhoff flow is unbounded. 
We may estimate the drift area using formula (4.4) for cases when the size of the wake is smaller than or comparable to the size of the cylinder (which is what we may expect in many practical situations). If we consider the range $\Gamma \in[0,7]$, we find that the relative difference between the total drift areas in the Föppl flow and in the wakeless potential flow, i.e., $D$ and $D_{1}$, is approximately $-7 \%$ when the drift area achieves its minimum (see Figure 4.5 ) and $65 \%$ when $\Gamma \approx 7$. In Figure 6.1 we can see that for the representative wakes shown in Figure $4.2 \mathrm{~b}-\mathrm{c}$, which are of relatively small size, $D_{1}$ may be a good approximation for the actual total drift area $D$. However, for values greater than $\Gamma \approx 4.5$, the relative difference exceeds $10 \%$. Therefore, in practice, when attached vortices are present and are large enough, it may be useful to take into consideration their effects on the drift area. We add that, as discussed in Introduction, this analysis is based on the idealized concept of the total drift area and in practical settings, depending on the actual travel times of the obstacle, it may be advisable to consider partial drift.

We expect that the results reported here may help improve the accuracy of modeling efforts concerning biogenic mixing, such as those reported in [7]. Calculation of quantities such as the mean squared displacement or the effective diffusivity as in $[3,8]$ for Föppl flow requires a careful consideration of the quantity of particles that interact with the body and the wake and may be considered as a useful subject of future studies. In addition, there are a number of open questions which may deserve further study concerning, for example, the drift induced by pairs or larger groups of moving obstacles (in the context of the potential flow theory, such flows can be studied using the formalism based on the Schottky-Klein function [42]), or obstacles 


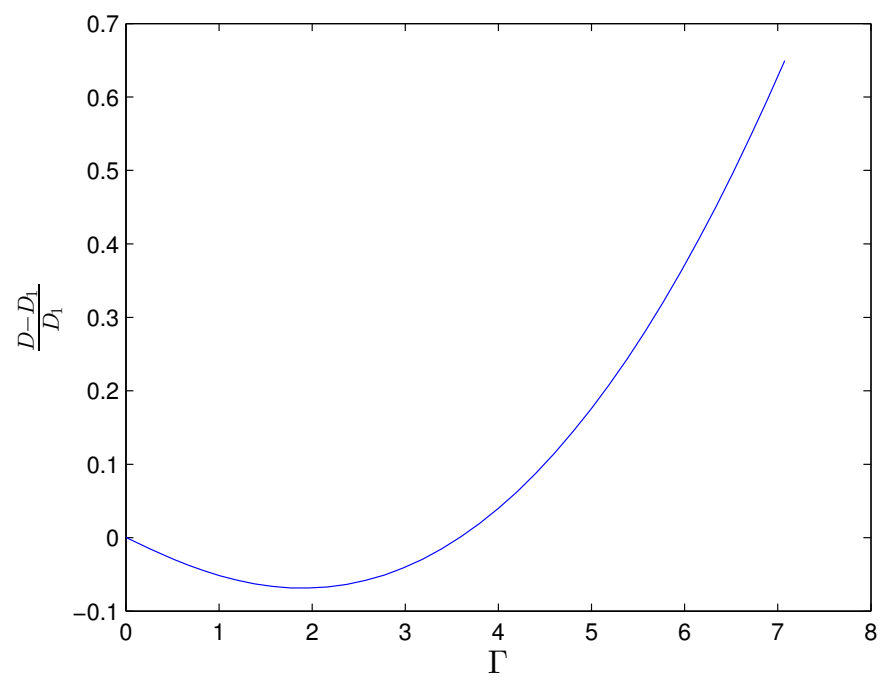

Figure 6.1: Relative difference between the drift area in the Föppl flow $(D)$ and in wakeless potential flow $\left(D_{1}\right)$ as a function of the circulation $\Gamma$.

with asymmetric wakes as were recently reported in [43]. The problem of identifying the shape of the obstacle which will produce a prescribed drift will lead to some interesting shape-optimization problems. 


\section{Appendix A}

\section{Added Mass}

Added mass is a concept related to objects that are in motion in a fluid. For instance, when calculating physical quantities such as the force, energy or momentum of a body immersed in a fluid, the added mass takes into account the additional effort that is required to not only push the body, but also the surrounding fluid which imposes an additional resistance. One area of application where the concept of added mass is particularly useful is in ocean engineering and naval construction [44]. For a general overview of added mass, see $[1,45,46]$. As discussed in Chapter 3.2, due to Darwin's theorem, the total drift area can be obtained by calculating the added mass. In this appendix, we will present an overview of added mass in the context of $2 \mathrm{D}$ potential flows which will provide a useful background in understanding how to calculate the total drift area according to equation (3.9) and (3.12).

Suppose that an object with mass $m$ which is immersed in a fluid has an acceleration a corresponding to some applied force $\mathbf{F}$. Since the object is surrounded by a fluid with density $\rho$, a part of the work applied to the system to accelerate the object is necessarily distributed amongst the fluid, which itself is also accelerated. To 
account for this effect, we may consider an equivalent system which is in a vacuum such that $\mathbf{F}=\left(m+m^{\prime}\right) \mathbf{a}$, where $m^{\prime} \in \mathbb{R}$ and $\left(m+m^{\prime}\right)$ is called the added mass. Thus, we see that by considering a system where the mass of the object is increased by some amount, $m^{\prime}$, we can take into account the additional effort that is required to accelerate an object in the presence of a fluid. For arbitrary geometries of the object, however, the added mass is not necessarily a single scalar quantity since it will depend on the direction of acceleration. That is, an acceleration in one direction may yield an added mass effect in another direction due to the specific object geometry. For this reason, in its most general case in three-dimensions, the added mass which we will denote $\mathbf{A}$, is a $6 \times 6$ tensor referred to as the added mass tensor with components $A_{\alpha \beta}$, where $\alpha, \beta=1,2,3$ refer to the linear components along the Cartesian coordinate system and $\alpha, \beta=4,5,6$ refer to the angular components along the Cartesian planes. Using the added mass tensor, we may write $\mathbf{F}=\mathbf{A a}$. In this thesis, we are interested in the 2D case with no body rotations such that we are limited to $\alpha, \beta=1,2$.

For an object in motion with velocity $\mathbf{U}=\left[U_{1}, U_{2}\right]^{T}, U_{1}, U_{2} \in \mathbb{R}$, in a potential fluid, there is a scalar potential $\phi$, cf. (1.8), which characterizes the flow. Since Laplace's equation (1.9) and the boundary condition (1.4) are linear, we may decompose the potential into components corresponding to the $x$ and $y$ directions. That is, we may introduce the vector potential $\phi:=\left[\phi_{1}, \phi_{2}\right]$, such that

$$
\phi=\mathbf{U} \phi=U_{1} \phi_{1}+U_{2} \phi_{2} .
$$


Then, the added mass tensor is given by

$$
A_{\alpha \beta}=-\rho \oint_{\mathcal{C}} \phi_{\alpha} \frac{\partial \phi_{\beta}}{\partial n} d s
$$

where $\mathcal{C}$ is a closed contour along the body which is in contact with the fluid and the

quantity $\frac{\partial \phi_{\beta}}{\partial n}$ denotes the normal derivative where the normal vector at the surface of the object, $\mathbf{n}=\left[n_{1}, n_{2}\right]^{T}$, points away from the object's interior. For a potential fluid, the added mass tensor is symmetric, such that $A_{\alpha \beta}=A_{\beta \alpha}[46]$. Due to the boundary condition (1.4), we may simplify (A.2) by writing

$$
\frac{\partial \phi_{\beta}}{\partial n}=\boldsymbol{\nabla} \phi_{\beta} \cdot \mathbf{n}=n_{\beta}
$$

Then, we get that the added mass is equal to

$$
A_{\alpha \beta}=-\rho \oint_{\mathcal{C}} \phi_{\alpha} n_{\beta} d s
$$

It should be noted that this is the origin of the formula (3.9) used when computing the added mass and total drift area for Föppl flow in Chapter 3.2.

As an example, we may consider the uniform motion in some arbitrary direction of a circular cylinder with unit radius in a fluid. First, to obtain the scalar potential $\phi$, we may start with the equation for the complex potential in uniform flow $W(z)=U e^{-i \alpha} z$ where $U$ is the flow speed and $\alpha$ is the direction. Using the Milne-Thomson Circle Theorem from Chapter 1.3 and after changing the frame of reference to where the fluid flow is zero at infinity, we get that the scalar potential for the moving cylinder 
is

$$
\phi=\frac{U \cos \alpha}{r} \cos \theta^{\prime}+\frac{U \sin \alpha}{r} \sin \theta^{\prime}
$$

Since $U$ was defined as the flow velocity, which is equal to the magnitude of the cylinder's velocity but opposite in its direction, we have that the object is in motion with velocity $\mathbf{U}=\left[U_{1}, U_{2}\right]^{T}=[-U \cos \alpha,-U \sin \alpha]^{T}$. Thus, now introducing the vector potential $\phi$, we have that

$$
\phi=U_{1}\left(-\frac{\cos \theta^{\prime}}{r}\right)+U_{2}\left(-\frac{\sin \theta^{\prime}}{r}\right)=\mathbf{U} \cdot \boldsymbol{\phi},
$$

where $\phi=\left[\left(-\frac{\cos \theta^{\prime}}{r}\right),\left(-\frac{\sin \theta^{\prime}}{r}\right)\right]$. Thus, using (A.4) with $\rho=1$, we find that the added mass tensor is equal to $\pi \mathbf{I}_{2}$ where $\mathbf{I}_{2}$ is the identity matrix of size 2 . Note that the added mass tensor does not depend on the particular velocity of the object.

In calculating the total drift area when the cylinder's motion is in the $x$ direction only, we are interested in the $A_{11}$ element of the added mass tensor. Note that the total drift area for the wakeless potential flow given by $D_{1}=\pi$ matches the result obtained in the example above. 


\section{Bibliography}

[1] S. Childress. An Introduction to Theoretical Fluid Mechanics. Courant Lecture Notes in Mathematics. American Mathematical Society, Courant Institute of Mathematical Sciences, 2009.

[2] I. Eames. Evolution of Darwin's concept of drift and application to multibody and multiphase flows. Philosophical Transactions of the Royal Society A: Mathematical, Physical and Engineering Sciences, pages 2951-2966, 2003.

[3] J.-L. Thiffeault and S. Childress. Stirring by swimming bodies. Physics Letters A, 374:3487-3490, 2010.

[4] C. Jung, T. Tél, and E. Ziemniak. Application of scattering chaos to particle transport in a hydrodynamical flow. Chaos, 3:555-568, 1993.

[5] E. Ziemniak, C. Jung, and T. Tél. Tracer dynamics in open hydrodynamical flows as chaotic scattering. Physica D, 76:123-146, 1994.

[6] W. H. Munk. Abyssal recipes. Deep-Sea Research, 13:707-730, 1966.

[7] K. Katija and J. O. Dabiri. A viscosity-enhanced mechanism for biogenic ocean mixing. Nature, 460:624-626, 2009. 
[8] D. O. Pushkin, H. Shum, and J. M. Yeomans. Fluid transport by individual microswimmers. Journal of Fluid Mechanics, 726:5-25, 2013.

[9] S. Melkoumian and B. Protas. Wake effects on drift in two-dimensional inviscid incompressible flows. Physics of Fluids, 26:123601, 2014.

[10] A. J. Chorin and J. E. Marsden. A Mathematical Introduction to Fluid Mechanics. Springer, 1993.

[11] L. M. Milne-Thomson. Theoretical Hydrodynamics. Dover, 1968.

[12] S. D. Fisher. Complex Variables. Wadsworth \& Brooks, Pacific Grove, CA, 1990.

[13] G.K. Batchelor. An Introduction to Fluid Mechanics. Cambridge University Press, Cambridge, UK, 2000.

[14] J. Clerk-Maxwell. On the displacement in a case of fluid motion. Proceedings of the London Mathematical Society, 3:82-87, 1870.

[15] Raph Levien. The elastica: a mathematical history. Technical Report UCB/EECS-2008-103, EECS Department, University of California, Berkeley, Aug 2008.

[16] George P. Thomson. Charles Galton Darwin. 1887-1962. Biographical Memoirs of Fellows of the Royal Society, 9:69-85, 1963.

[17] Ch. Darwin. Note on hydrodynamics. Mathematical Proceedings of the Cambridge Philosophical Society, 49:342-354, 1953.

[18] C.-S. Yih. New derivations of Darwin's theorem. Journal of Fluid Mechanics, 152:163-172, 1985. 
[19] T. B. Benjamin. Note on added mass and drift. Journal of Fluid Mechanics, 169:251-256, 1986.

[20] C.-S. Yih. Evolution of Darwinian drift. Journal of Fluid Mechanics, 347:1-11, 1997.

[21] I. Eames, S. E. Belcher, and J. C. R. Hunt. Drift, partial drift and Darwin's proposition. Journal of Fluid Mechanics, 275:201-223, 1994.

[22] J. Bataille, M. Lance, and J. L. Marie. Some Aspects of the Modeling of Bubbly Flows. In G. F. Hewitt, F. Mayinger, and J. R. Riznic, editors, Phase-interface phenomena in multiphase flow, Proceedings of the International Centre for Heat and Mass Transfer, pages 179-193. Hemisphere Pub. Corp., New York, 1991.

[23] I. Eames and M. E. McIntyre. On the connection between stokes drift and darwin drift. Mathematical Proceedings of the Cambridge Philosophical Society, 126:171-174, 1999.

[24] J. O. Dabiri. Nore on the induced Lagrangian drift and added-mass of a vortex. Journal of Fluid Mechanics, 547:105-113, 2006.

[25] R. Camassa, R. M. McLaughlin, M. N. J. Moore, and A. Vaidya. Brachistochrones in potential flow and the connection to Darwin's theorem. Physics Letters A, 372:6742-6749, 2008.

[26] Milton Van Dyke. An Album of Fluid Motion. The Parabolic Press, Stanford, CA, 1988.

[27] V. V. Sychev, A. I. Ruban, V. V. Sychev, and G. L. Korolev. Asymptotic Theory of Separated Flows. Cambridge University Press, 1998. 
[28] L. Föppl. Wirbelbewegung hinter einem Kreiscylinder. Sitzb. d. k. Bayr. Akad. d. Wiss., 1:1-17, 1913.

[29] A. Elcrat, B. Fornberg, M. Horn, and K. Miller. Some steady vortex flows past a circular cylinder. J. Fluid Mech., 409:13-27, 2000.

[30] B. Protas. Higher-order Föppl models of steady wake flows. Physics of Fluids, 11:117109, 2006.

[31] S. Tang and N. Aubry. On the symmetry breaking instability leading to vortex shedding. Phys. Fluids, 9:2550-2561, 1997.

[32] F. Li and N. Aubry. Feedback control of a flow past a cylinder via transverse motion. Physics of Fluids, 15:2163-2176, 2003.

[33] B. Protas. Linear feedback stabilization of laminar vortex shedding based on a point vortex model. Physics of Fluids, 16:4473-4488, 2004.

[34] B. Protas. Center manifold analysis of a point-vortex model of vortex shedding with control. Physica D, 228:179-187, 2007.

[35] B. Protas. Vortex dynamics models in flow control problems. Nonlinearity, 21:R203-R250, 2008. (invited paper).

[36] T. Levi-Civita. Scie e leggi di reistenza. Rendiconti del Circolo Matematico di Palermo, XXIII:1-37, 1907.

[37] S. Brodetsky. Discontinuous fluid motion past circular and elliptic cylinders. Proceedings of the Royal Society of London. Series A, 102:542-553, 1923. 
[38] G. I. Taylor. The energy of a body moving in an infinite fluid, with an application to airships. Proceedings of the Royal Society A, 120:13, 1928.

[39] L. Landweber and C.-S. Yih. Forces, moments, and added masses for rankine bodies. Journal of Fluid Mechanics, 1:319-336, 1956.

[40] IEEE standard for floating-point arithmetic, August 2008. IEEE. doi:10.1109/IEEESTD.2008.4610935.

[41] I. Eames, D. Gobby, and S. B. Dalziel. Fluid displacement by Stokes flow past a spherical droplet. Journal of Fluid Mechanics, 485:67-85, 2003.

[42] D.G. Crowdy. Analytical solutions for uniform potential flow past multiple cylinders. Eur. J. Mech. B/Fluids, 25:459-470, 2006.

[43] A. Elcrat, M. Ferlauto, and L. Zannetti. Point vortex model for asymmetric inviscid wakes past bluff bodies. Fluid Dynamics Research, 46:031407, 2014.

[44] C. E. Brennen. A Review of Added Mass and Fluid Inertial Forces. Technical Report Report Number CR 82.010, Contract Number N62583-81-MR-554, Naval Civil Engineering Laboratory, Department of the Navy, Port Hueneme, CA 93043, January 1982.

[45] P.G. Saffman. Vortex Dynamics. University of Cambridge Press, 1992.

[46] Chia-Shun Yih. Fluid Mechancis: A Concise Introduction to the Theory. West River Press, Ann Arbor, Michigan, U.S.A., 1988. 